\title{
Dynamic Covalent Assembly of Peptoid-Based Ladder Oligomers by Vernier Templating
}

Tao Wei, ${ }^{\dagger}$ Jae Hwan Jung, ${ }^{\dagger}$ and Timothy F. Scott $*, \dagger, \ddagger$

${ }^{\dagger}$ Department of Chemical Engineering, University of Michigan, Ann Arbor, MI 48109

${ }^{\star}$ Macromolecular Science and Engineering, University of Michigan, Ann Arbor, MI 48109

\section{General Experimental Procedure}

All chemicals and reagents, unless specified, were purchased from commercial sources and used as received without any further purification. ${ }^{1} \mathrm{H}$ and ${ }^{13} \mathrm{C}$ NMR spectra were collected using Varian MR400 and Varian VNMRS 700 spectrometers. Chemical shifts were measured in $\delta$ (ppm) relative to residual solvent signals as internal standards $\left(\mathrm{CDCl}_{3}: 7.24\right.$ for ${ }^{1} \mathrm{H}, 77.23$ for ${ }^{13} \mathrm{C} ; \mathrm{CD}_{3} \mathrm{OD}: 4.78$ and 3.31 for ${ }^{1} \mathrm{H}$, 49.15 for ${ }^{13} \mathrm{C}$ ). Matrix-assisted laser desorption/ionization (MALDI) mass spectra were recorded using a Bruker Autoflex mass spectrometer, whereas electrospray ionization (ESI) mass spectra were recorded using an Agilent Q-TOF 1200 series spectrometer. MALDI analyses were performed in reflectron position ion mode using 2-(4-hydroxyphenylazo)benzoic acid (HABA) as the matrix, where $3 \mu \mathrm{L}$ of a solution of the sample in chloroform $(1.5 \mathrm{mM})$ was mixed with $10 \mu \mathrm{L}$ of a mixture of $6 \mathrm{mg}$ matrix in 300 $\mu \mathrm{L}$ acetonitrile, spotted on a MALDI sample plate (Bruker), and allowed to air dry. Reverse phase high performance liquid chromatography (RP-HPLC) was performed using a Shimadzu LC-6AD HPLC pump, equipped with a Shimadzu FRC 70A fraction collector, using analytical and preparative reversed phase Phenomenex Luna $\mathrm{C} 18$ (2) columns with a linear gradient of water and acetonitrile as the eluent at $30^{\circ} \mathrm{C}$, and monitored with a Shimadzu Prominence UV/vis detector at $214 \mathrm{~nm}$. Analytical gel permeation chromatography (GPC) was similarly performed using a Shimadzu LC-6AD HPLC pump, equipped with a series of three Phenogel GPC/SEC columns (length $300 \mathrm{~mm} \times$ diameter $7.8 \mathrm{~mm}$, pore sizes of 500, 100, and $50 \AA$ ) with $94: 4: 2(\mathrm{v} / \mathrm{v} / \mathrm{v}) \mathrm{CHCl}_{3}: \mathrm{MeOH}_{\mathrm{Et}} \mathrm{N}$ as the eluent at $30^{\circ} \mathrm{C}$, and monitored with a Shimadzu Prominence UV/vis detector at $313 \mathrm{~nm}$. The analytical GPC was calibrated utilizing low dispersity polystyrene standards (Polystyrene Standard (Low Molecular) Readycal Set, Fluka). 


\section{Abbreviations}

DMF: N,N'-dimethylformamide

DIC: N,N'-diisopropylcarbodiimide

DCM: dichloromethane

$\mathrm{Et}_{3} \mathrm{~N}$ : triethylamine

$\mathrm{Et}_{2} \mathrm{O}$ : diethyl ether

$\mathrm{LiAlH}_{4}$ : lithium aluminum hydride

$\mathrm{MeCN}$ : acetonitrile

NMP: N-methyl-2-pyrrolidone

TFA: trifluoroacetic acid

THF: tetrahydrofuran

\section{Monomer synthesis}

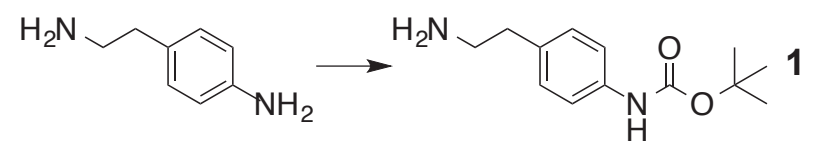

Scheme S1. Synthesis of 4-(2-aminoethyl)- $N$-(tert-butoxycarbonyl)phenylamine (1). Reagents and conditions: ditert-butyl dicarbonate, $10 \%$ acetic acid in water, 1,4-dioxane, r.t. overnight. ${ }^{[1]}$

4-(2-Aminoethyl)- $N$-(tert-butoxycarbonyl)phenylamine (1). The method to synthesize compound 1 was adapted from a published approach. ${ }^{[1]}$ Di-tert-butyl dicarbonate $(10 \mathrm{~g}, 45.8 \mathrm{mmol})$ in $200 \mathrm{~mL}$ 1,4-dioxane was added into to a solution of 4-(2-aminoethyl)aniline $(6 \mathrm{~g}, 44.1 \mathrm{mmol})$ in $200 \mathrm{~mL} \mathrm{10 \%}$ aq. acetic acid. The reaction mixture was stirred at room temperature overnight and then diluted with $600 \mathrm{~mL}$ deionized (DI) water and washed with $\mathrm{Et}_{2} \mathrm{O}(300 \mathrm{~mL})$ three times. The aqueous phase was adjusted to $\mathrm{pH} 14$ by addition of $2 \mathrm{M} \mathrm{NaOH}(\mathrm{aq})$ and was extracted with $\mathrm{Et}_{2} \mathrm{O}(150 \mathrm{~mL})$ three times. The combined organic layer was washed with DI water three times, dried over $\mathrm{Na}_{2} \mathrm{SO}_{4}$, filtered, and evaporated to dryness to yield 1 as a light yellow solid (7.0 g, 67\%).

${ }^{1} \mathrm{H}$ NMR (400 MHz, CD $\mathrm{CD}_{3}$ ) $\delta: 7.32$ (d, $\left.J=8.4,2 \mathrm{H}, \mathrm{Ar}\right), 7.11$ (d, $\left.J=8.4,2 \mathrm{H}, \mathrm{Ar}\right), 2.81-2.85$ (m, 2H, $\left.\mathrm{CH}_{2}-\mathrm{NH}_{2}\right), 2.69\left(\mathrm{t}, J=7.2,2 \mathrm{H},-\mathrm{CH}_{2}-\mathrm{Ar}\right), 1.51\left(\mathrm{~s}, 9 \mathrm{H},\left(-\mathrm{CH}_{3}\right)_{3}\right)$.

${ }^{13} \mathrm{C}$ NMR (100 MHz, CD $\left.\mathrm{CD}_{3} \mathrm{OD}\right) \delta: 155.41$ (1C, $\left.-\mathrm{C}=\mathrm{O}\right), 138.72$ (1C, Ar), 135.22 (1C, Ar), 130.04 (2C, Ar), $120.13(2 \mathrm{C}, \mathrm{Ar}), 80.72\left(1 \mathrm{C},-C\left(\mathrm{CH}_{3}\right)_{3}\right), 44.27\left(1 \mathrm{C},-\mathrm{CH}_{2}-\mathrm{NH}_{2}\right), 39.48\left(1 \mathrm{C},-\mathrm{CH}_{2}-\mathrm{Ar}\right), 28.72\left(3 \mathrm{C},\left(-\mathrm{CH}_{3}\right)_{3}\right)$. MS (ESI+): calcd for $\mathrm{C}_{13} \mathrm{H}_{20} \mathrm{~N}_{2} \mathrm{O}_{2}:[\mathrm{M}+\mathrm{H}]^{+}=237.1598[\mathrm{M}+\mathrm{Na}]^{+}=259.1417$, found: $\mathrm{m} / z=237.1595$ $[\mathrm{M}+\mathrm{H}]^{+}, m / z=259.1415[\mathrm{M}+\mathrm{Na}]^{+}$. 


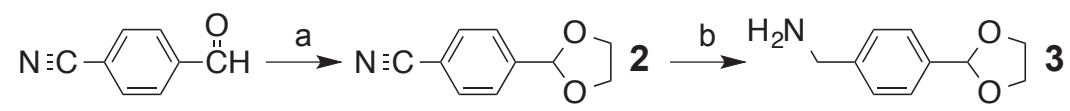

Scheme S2. Synthesis of 4-(1,3-dioxacyclopent-2-yl)benzylamine (3). Reagents and conditions: (a) ethylene glycol, toluene- $p$-sulfonic acid, toluene, reflux; (b) $\mathrm{LiAlH}_{4}, \mathrm{Et}_{2} \mathrm{O}, 0^{\circ} \mathrm{C}$ for $4 \mathrm{~h}$ then r.t. for $12 \mathrm{~h} .^{[2]}$

4-(1,3-Dioxacyclopent-2-yl)benzonitrile (2). The method to synthesize compound 2 was adapted from a published approach. ${ }^{[2]}$ Ethylene glycol $(42.2 \mathrm{~mL}, 0.768 \mathrm{~mol})$ and toluene-p-sulfonic acid $(0.02 \mathrm{~g}, 0.10$ mmol) were added into a solution of 4-cyanobenzaldehyde $(25 \mathrm{~g}, 0.190 \mathrm{~mol})$ in $200 \mathrm{~mL}$ of toluene. The reaction mixture was stirred and refluxed overnight. Azeotropic distillation with a Dean-Stark trap was used to remove water generated during the reaction. The reaction mixture was then cooled to room temperature and $40 \mathrm{~mL}$ of a $5 \% \mathrm{NaHCO}_{3}$ aqueous solution was added. The organic layer was extracted, washed with DI water three times, and dried over $\mathrm{Na}_{2} \mathrm{SO}_{4}$. The solvent was subsequently evaporated to dryness under reduced pressure to yield 2 as a white crystalline solid (28.75 g, 86.4\%).

${ }^{1} \mathrm{H}$ NMR (400 MHz, $\left.\mathrm{CDCl}_{3}\right) \delta: 7.67$ (d, $\left.J=8.0,2 \mathrm{H}, \mathrm{Ar}\right), 7.59$ (d, $\left.J=8.4,2 \mathrm{H}, \mathrm{Ar}\right), 5.84(\mathrm{~s}, 1 \mathrm{H}, \mathrm{CH}), 4.12$ - $4.03\left(\mathrm{AA}^{\prime} \mathrm{BB}^{\prime}, 4 \mathrm{H},\left(\mathrm{CH}_{2} \mathrm{O}\right)_{2}\right)$.

${ }^{13} \mathrm{C}$ NMR (100 MHz, $\mathrm{CDCl}_{3}$ ) $\delta: 143.20$ (1C, Ar), 132.34 (2C, Ar), 127.30 (2C, Ar), 118.72 (1C, CN), $113.02(1 \mathrm{C}, \mathrm{Ar}), 102.56(1 \mathrm{C}, \mathrm{CH}), 65.57\left(2 \mathrm{C},-\mathrm{CH}_{2} \mathrm{O}-\right)$.

4-(1,3-Dioxacyclopent-2-yl)benzylamine (3). The method to synthesize compound $\mathbf{3}$ was adapted from a published approach. ${ }^{[2]} \mathrm{A}$ solution of compound $2(10 \mathrm{~g}, 0.057 \mathrm{~mol})$ in $100 \mathrm{~mL}$ of dry $^{\mathrm{Et}} \mathrm{t}_{2} \mathrm{O}$ was added dropwise into a well-mixed suspension of $\mathrm{LiAlH}_{4}(4.33 \mathrm{~g}, 0.114 \mathrm{~mol})$ in $100 \mathrm{~mL}$ of dry $\mathrm{Et}_{2} \mathrm{O}$ at $0^{\circ} \mathrm{C}$ under nitrogen. The reaction mixture was stirred for $4 \mathrm{~h}$ at $0^{\circ} \mathrm{C}$ and $12 \mathrm{~h}$ at room temperature, then quenched by $95 \%$ ethanol $(30 \mathrm{~mL})$, and further quenched with $50 \%$ ethanol in water $(20 \mathrm{~mL})$. The ether supernatant was separated, evaporated to dryness under reduced pressure and the product redissolved in ether. The solvent was then washed twice with DI water, dried over sodium sulfate, and removed to afford a yellow oil. The oil was purified on a silica gel column, initially flushed with ethyl acetate and subsequently eluted with a 90:10 to 70:30 ethyl acetate:methanol gradient. Solvent removal yielded compound $\mathbf{3}$ as a light yellow oil $(7.20 \mathrm{~g}, 70.4 \%)$.

${ }^{1} \mathrm{H}$ NMR (400 MHz, $\left.\mathrm{CDCl}_{3}\right) \delta: 7.44(\mathrm{~d}, J=8.0,2 \mathrm{H}, \mathrm{Ar}), 7.32$ (d, $\left.J=8.0,2 \mathrm{H}, \mathrm{Ar}\right), 5.80(\mathrm{~s}, 1 \mathrm{H}, \mathrm{CH}), 4.14$ - $4.0\left(\mathrm{AA}^{\prime} \mathrm{BB}^{\prime}, 4 \mathrm{H},\left(\mathrm{CH}_{2} \mathrm{O}\right)_{2}\right), 3.87\left(\mathrm{~s}, 2 \mathrm{H},-\mathrm{CH}_{2}-\mathrm{NH}_{2}\right)$.

${ }^{13} \mathrm{C}$ NMR (100 MHz, $\left.\mathrm{CDCl}_{3}\right) \delta: 144.53$ (1C, Ar), 136.53 (1C, Ar), 127.16 (2C, Ar), 126.77 (2C, Ar), $103.72(1 \mathrm{C}, \mathrm{CH}), 65.39\left(2 \mathrm{C},-\mathrm{CH}_{2} \mathrm{O}-\right), 46.35\left(1 \mathrm{C},-\mathrm{CH}_{2}-\mathrm{NH}_{2}\right)$.

MS (ESI+): calcd for $\mathrm{C}_{10} \mathrm{H}_{13} \mathrm{NO}_{2}:[\mathrm{M}+\mathrm{H}]^{+}=180.1019$, found: $m / z=180.1016[\mathrm{M}+\mathrm{H}]^{+}$. 


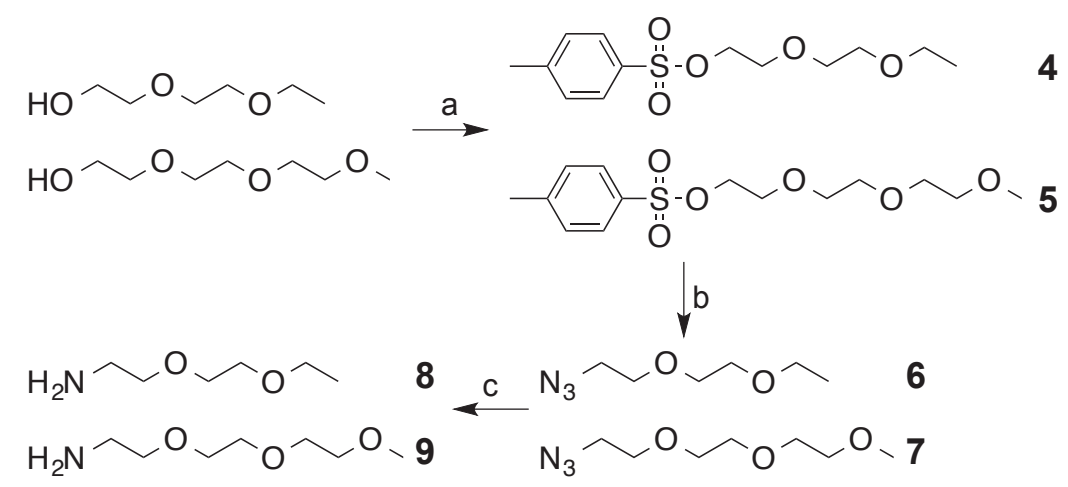

Scheme S3. Synthesis of 2-(2-ethoxyethoxy)ethylamine $\left(\mathrm{E}^{3} \mathrm{~A}\right)(\mathbf{8})$ and 2-(2-(2-methoxyethoxy)ethoxy)ethylamine $\left(\mathrm{ME}^{3} \mathrm{~A}\right)(9)$. Reagents and conditions: (a) tosyl chloride, $\mathrm{THF}, 0^{\circ} \mathrm{C}$, (b) $\mathrm{NaN}_{3}, \mathrm{DMF}, 60^{\circ} \mathrm{C}, 36 \mathrm{~h}$, and (c) $\mathrm{TPP}$, water. $^{[3]}$

2-(2-Ethoxyethoxy)ethyl tosylate (4). The method to synthesize compound 4 was adapted from a published approach. ${ }^{[3]}$ Diethylene glycol monoethyl ether $(20 \mathrm{~g}, 0.15 \mathrm{~mol})$ and $50 \mathrm{ml}$ of THF were charged to a $500 \mathrm{~mL}$ round bottom flask with a magnetic stirrer. This reaction mixture was cooled to $0^{\circ} \mathrm{C}$ and $50 \mathrm{ml}$ of $6 \mathrm{M} \mathrm{NaOH}$ was added, followed by dropwise addition of tosyl chloride (54 g, $0.28 \mathrm{~mol})$ in $80 \mathrm{ml}$ THF under $\mathrm{N}_{2}$. After stirring for $1 \mathrm{~h}$ at $0^{\circ} \mathrm{C}$, the reaction mixture was allowed to reach room temperature and stirred for another $1 \mathrm{~h}$. The resulting mixture was extracted with $\mathrm{Et}_{2} \mathrm{O}(400 \mathrm{ml})$ and the organic layer was washed with $1 \mathrm{M} \mathrm{NaOH}$ and water. After drying over $\mathrm{MgSO}_{4}$, the solution was evaporated under vacuum to yield $\mathbf{4}$ as a colorless liquid (42 g, 98\%).

${ }^{1} \mathrm{H}$ NMR $\left(400 \mathrm{MHz}, \mathrm{CDCl}_{3}\right) \delta: 7.78(\mathrm{~d}, J=8.0,2 \mathrm{H},-\mathrm{S}-\mathrm{C}=\mathrm{CH}-\mathrm{CH}), 7.33(\mathrm{~d}, J=8.5,2 \mathrm{H},-\mathrm{S}-\mathrm{C}=\mathrm{CH}-\mathrm{CH})$, $4.15\left(\mathrm{t}, J=5.0,2 \mathrm{H},-\mathrm{CH}_{2}-\mathrm{CH}_{2}-\mathrm{O}-\mathrm{Ts}\right), 3.68$ (t, $\left.J=5.0,2 \mathrm{H}, \mathrm{CH}_{2}-\mathrm{CH}_{2}-\mathrm{O}-\mathrm{Ts}\right), 3.60-3.42$ (m, 6H, O-CH $2^{-}$ $\mathrm{CH}_{2}-\mathrm{O}-\mathrm{CH}_{2}-\mathrm{CH}_{3}$ ), 2.43 (s, 3H, C-CH3), 1.17 (t, $J=7.0,3 \mathrm{H}, \mathrm{O}-\mathrm{CH}_{2}-\mathrm{CH}_{3}$ ).

${ }^{13} \mathrm{C}$ NMR $\left(100 \mathrm{MHz}, \mathrm{CDCl}_{3}\right) \delta: 144.79$ (1C, -S-C $_{q}$-Ar), 132.95 (1C, $\mathrm{C}_{p}$-Ar), $130.26\left(1 \mathrm{C}, \mathrm{C}_{m}-\mathrm{Ar}\right), 129.80$ $\left(1 \mathrm{C}, \mathrm{C}_{m}-\mathrm{Ar}\right), 127.90$ (1C, $\left.\mathrm{C}_{o}-\mathrm{Ar}\right), 126.95$ (1C, $\left.\mathrm{C}_{o}-\mathrm{Ar}\right), 70.75\left(1 \mathrm{C}, \mathrm{O}-\mathrm{CH}_{2}-\mathrm{CH}_{2}-\mathrm{O}_{-} \mathrm{CH}_{2}-\mathrm{CH}_{3}\right), 69.68$ (1C, O- $\left.\mathrm{CH}_{2}-\mathrm{CH}_{2}-\mathrm{O}-\mathrm{CH}_{2}-\mathrm{CH}_{3}\right), 69.29$ (1C, $\left.-\mathrm{CH}_{2}-\mathrm{CH}_{2}-\mathrm{O}-\mathrm{Ts}\right), 68.61$ (1C, $\left.-\mathrm{CH}_{2}-\mathrm{CH}_{2}-\mathrm{O}-\mathrm{Ts}\right), 66.57$ (1C, O-CH $\left.\mathrm{CH}_{3}\right), 21.56\left(1 \mathrm{C}, \mathrm{C}-\mathrm{CH}_{3}\right), 15.11\left(1 \mathrm{C}, \mathrm{O}-\mathrm{CH}_{2}-\mathrm{CH}_{3}\right)$.

2-(2-(2-Methoxyethoxy)ethoxy)ethyl tosylate (5). Triethylene glycol monomethyl ether (24.6 g, 0.15 mol) was allowed to react according to the above procedure, yield: $47 \mathrm{~g}(98 \%)$.

${ }^{1} \mathrm{H}$ NMR $\left(400 \mathrm{MHz}, \mathrm{CDCl}_{3}\right) \delta: 7.77(\mathrm{~d}, J=8.0,2 \mathrm{H},-\mathrm{S}-\mathrm{C}=\mathrm{CH}-\mathrm{CH}), 7.32(\mathrm{~d}, J=8.0,2 \mathrm{H},-\mathrm{S}-\mathrm{C}=\mathrm{CH}-\mathrm{CH})$, 4.13 (t, $\left.J=5.0,2 \mathrm{H},-\mathrm{CH}_{2}-\mathrm{CH}_{2}-\mathrm{O}-\mathrm{Ts}\right), 3.66$ (t, $\left.J=4.0,2 \mathrm{H}, \mathrm{CH}_{2}-\mathrm{CH}_{2}-\mathrm{O}-\mathrm{Ts}\right), 3.57$ (m, 6H, O-CH2 $-\mathrm{CH}_{2}-\mathrm{O}-$ $\left.\mathrm{CH}_{2}-\mathrm{CH}_{2}-\mathrm{O}-\mathrm{CH}_{3}\right), 3.50\left(\mathrm{~m}, 2 \mathrm{H}, \mathrm{O}-\mathrm{CH}_{2}-\mathrm{CH}_{2}-\mathrm{O}-\mathrm{CH}_{3}\right), 3.35$ (s, 3H, O-CH3 $), 2.44\left(\mathrm{~s}, 3 \mathrm{H}, \mathrm{C}-\mathrm{CH}_{3}\right)$.

${ }^{13} \mathrm{C}$ NMR (100 MHz, $\left.\mathrm{CDCl}_{3}\right) \delta: 144.78$ (1C, $\left.-\mathrm{S}-\mathrm{C}_{q}-\mathrm{Ar}\right), 132.95$ (1C, $\mathrm{C}_{p}$-Ar), $130.26\left(1 \mathrm{C}, \mathrm{C}_{m}-\mathrm{Ar}\right), 129.80$ (1C, $\mathrm{C}_{m}$-Ar), 127.88 (1C, $\left.\mathrm{C}_{o}-\mathrm{Ar}\right), 126.93$ (1C, $\left.\mathrm{C}_{o}-\mathrm{Ar}\right), 71.83\left(1 \mathrm{C}, \mathrm{O}-\mathrm{CH}_{2}-\mathrm{CH}_{2}-\mathrm{O}-\mathrm{CH}_{3}\right), 70.64$ (1C, O- 
$\left.\mathrm{CH}_{2}-\mathrm{CH}_{2}-\mathrm{O}-\mathrm{CH}_{3}\right), 70.45\left(2 \mathrm{C}, \mathrm{O}-\mathrm{CH}_{2}-\mathrm{CH}_{2}-\mathrm{O}-\mathrm{CH}_{2}-\mathrm{CH}_{2}-\mathrm{O}-\mathrm{CH}_{3}\right), 69.28\left(1 \mathrm{C},-\mathrm{CH}_{2}-\mathrm{CH}_{2}-\mathrm{O}-\mathrm{Ts}\right), 68.56(1 \mathrm{C}$, $\left.-\mathrm{CH}_{2}-\mathrm{CH}_{2}-\mathrm{O}-\mathrm{Ts}\right), 58.90\left(1 \mathrm{C}, \mathrm{O}-\mathrm{CH}_{3}\right), 21.56\left(1 \mathrm{C}, \mathrm{C}-\mathrm{CH}_{3}\right)$.

2-(2-Ethoxyethoxy)ethyl azide (6). The method to synthesize compound 6 was adapted from a published approach. ${ }^{[3]}$ A $500 \mathrm{~mL}$ round bottom flask was charged with compound 4 (40 g, $\left.0.14 \mathrm{~mol}\right)$ and DMF (250 ml) under $\mathrm{N}_{2}$. To this mixture, $\mathrm{NaN}_{3}(31.5 \mathrm{~g}, 3.5 \mathrm{eq}, 0.49 \mathrm{~mol})$ was added. The reaction mixture was heated at $60^{\circ} \mathrm{C}$ for $36 \mathrm{~h}$ and then cooled to room temperature. The reaction mixture was diluted with a large amount of water and extracted with $\mathrm{Et}_{2} \mathrm{O}$. The organic layer was washed with water and dried over $\mathrm{MgSO}_{4}$, and evaporated under vacuum to afford compound 6 as a yellow liquid (19 $\left.\mathrm{g}, 85 \%\right)$.

${ }^{1} \mathrm{H}$ NMR (400 MHz, $\mathrm{CDCl}_{3}$ ) $\delta: 3.64\left(\mathrm{~m}, 4 \mathrm{H}, \mathrm{O}-\mathrm{CH}_{2}-\mathrm{CH}_{2}-\mathrm{O}\right), 3.58\left(\mathrm{~m}, 2 \mathrm{H}, \mathrm{N}_{3}-\mathrm{CH}_{2}-\mathrm{CH}_{2}-\mathrm{O}\right), 3.51$ (q, $J=$ 7.5, 2H, O-CH $\mathrm{CH}_{2}-\mathrm{CH}_{3}$ ), 3.38 (t, $\left.J=5.0,2 \mathrm{H}, \mathrm{N}_{3}-\mathrm{CH}_{2}-\mathrm{CH}_{2}-\mathrm{O}\right), 1.19$ (t, $J=7.5,3 \mathrm{H}, \mathrm{O}_{-} \mathrm{CH}_{2}-\mathrm{CH}_{3}$ ).

${ }^{13} \mathrm{C}$ NMR $\left(100 \mathrm{MHz}, \mathrm{CDCl}_{3}\right) \delta: 70.70\left(1 \mathrm{C}, \mathrm{N}_{3}-\mathrm{CH}_{2}-\mathrm{CH}_{2}-\mathrm{O}\right), 69.97\left(1 \mathrm{C}, \mathrm{O}-\mathrm{CH}_{2}-\mathrm{CH}_{2}-\mathrm{O}-\mathrm{CH}_{2}-\mathrm{CH}_{3}\right), 69.80$ $\left(1 \mathrm{C}, \mathrm{O}-\mathrm{CH}_{2}-\mathrm{CH}_{2}-\mathrm{O}-\mathrm{CH}_{2}-\mathrm{CH}_{3}\right), 66.63\left(1 \mathrm{C}, \mathrm{O}-\mathrm{CH}_{2}-\mathrm{CH}_{3}\right), 50.60\left(1 \mathrm{C}, \mathrm{N}_{3}-\mathrm{CH}_{2}-\mathrm{CH}_{2}-\mathrm{O}\right), 15.08$ (1C, O-CH $\left.\mathrm{CH}_{3}\right)$.

2-(2-(2-Methoxyethoxy)ethoxy)ethyl azide (7). Following the procedure given for the synthesis of 6, this compound was prepared from compound 5 (44.6 g, $0.14 \mathrm{~mol})$, yield: $22 \mathrm{~g}$ (83\%).

${ }^{1} \mathrm{H}$ NMR (400 MHz, $\left.\mathrm{CDCl}_{3}\right) \delta: 3.63\left(\mathrm{~m}, 8 \mathrm{H}, \mathrm{O}-\mathrm{CH}_{2}-\mathrm{CH}_{2}-\mathrm{O}-\mathrm{CH}_{2}-\mathrm{CH}_{2}-\mathrm{O}-\mathrm{CH}_{3}\right), 3.52\left(\mathrm{~m}, 2 \mathrm{H}, \mathrm{N}_{3}-\mathrm{CH}_{2}-\right.$ $\left.\mathrm{CH}_{2}-\mathrm{O}\right), 3.35$ (m, 5H, O- $\mathrm{CH}_{3}$ and $\left.\mathrm{N}_{3}-\mathrm{CH}_{2}-\mathrm{CH}_{2}-\mathrm{O}\right)$.

${ }^{13} \mathrm{C}$ NMR (100 MHz, $\left.\mathrm{CDCl}_{3}\right) \delta: 71.83\left(1 \mathrm{C}, \mathrm{O}-\mathrm{CH}_{2}-\mathrm{CH}_{2}-\mathrm{O}-\mathrm{CH}_{3}\right), 70.57\left(2 \mathrm{C}, \mathrm{O}-\mathrm{CH}_{2}-\mathrm{CH}_{2}-\mathrm{O}-\mathrm{CH}_{2}-\mathrm{CH}_{2}-\mathrm{O}-\right.$ $\left.\mathrm{CH}_{3}\right), 70.49\left(1 \mathrm{C}, \mathrm{O}-\mathrm{CH}_{2}-\mathrm{CH}_{2}-\mathrm{O}-\mathrm{CH}_{3}\right), 69.93\left(1 \mathrm{C}, \mathrm{N}_{3}-\mathrm{CH}_{2}-\mathrm{CH}_{2}-\mathrm{O}\right), 58.87\left(1 \mathrm{C}, \mathrm{O}-\mathrm{CH}_{3}\right), 50.58\left(1 \mathrm{C}, \mathrm{N}_{3}-\right.$ $\left.\mathrm{CH}_{2}-\mathrm{CH}_{2}-\mathrm{O}\right)$.

2-(2-Ethoxyethoxy)ethylamine $\left(\mathbf{E}^{\mathbf{3}} \mathbf{A}\right)(\mathbf{8})$. The method to synthesize compound $\mathbf{8}$ was adapted from a published approach. ${ }^{[3]}$ A $250 \mathrm{~mL}$ three necked round bottom flask equipped with magnetic stirrer was charged with compound $6(20 \mathrm{~g}, 0.13 \mathrm{mmol})$ and THF $(160 \mathrm{ml})$. Triphenylphosphine (TPP) (40 g, 0.15 mol, $1.1 \mathrm{eq}$ ) was then added and stirred overnight at room temperature under $\mathrm{N}_{2}$. The reaction mixture was quenched with water $(220 \mathrm{~mL})$, and allowed to stir for another day. The resulting solution was washed with toluene and DCM, and evaporated under vacuum to yield compound $\mathbf{8}$ as a yellow liquid (10 g, 58\%).

${ }^{1} \mathrm{H}$ NMR $\left(400 \mathrm{MHz}, \mathrm{CDCl}_{3}\right) \delta: 3.62-3.42\left(\mathrm{~m}, 8 \mathrm{H}, \mathrm{NH}_{2}-\mathrm{CH}_{2}-\mathrm{CH}_{2}-\mathrm{O}-\mathrm{CH}_{2}-\mathrm{CH}_{2}-\mathrm{O}-\mathrm{CH}_{2}-\mathrm{CH}_{3}\right), 2.82(\mathrm{~m}$, $\left.2 \mathrm{H}, \mathrm{NH}_{2}-\mathrm{CH}_{2}-\mathrm{CH}_{2}-\mathrm{O}\right), 1.48$ (s, 2H, $\left.\mathrm{NH}_{2}\right), 1.16$ (t, $\left.J=7.5,3 \mathrm{H}, \mathrm{O}-\mathrm{CH}_{2}-\mathrm{CH}_{3}\right)$. 
${ }^{13} \mathrm{C}$ NMR (100 MHz, $\left.\mathrm{CDCl}_{3}\right) \delta: 73.14\left(1 \mathrm{C}, \mathrm{NH}_{2}-\mathrm{CH}_{2}-\mathrm{CH}_{2}-\mathrm{O}\right), 70.72\left(1 \mathrm{C}, \mathrm{O}-\mathrm{CH}_{2}-\mathrm{CH}_{2}-\mathrm{O}-\mathrm{CH}_{2}-\mathrm{CH}_{3}\right)$, $69.64\left(1 \mathrm{C}, \mathrm{O}-\mathrm{CH}_{2}-\mathrm{CH}_{2}-\mathrm{O}-\mathrm{CH}_{2}-\mathrm{CH}_{3}\right), 66.45\left(1 \mathrm{C}, \mathrm{O}-\mathrm{CH}_{2}-\mathrm{CH}_{3}\right), 41.35$ (1C, $\left.\mathrm{NH}_{2}-\mathrm{CH}_{2}-\mathrm{CH}_{2}-\mathrm{O}\right), 15.00$ (1C, $\left.\mathrm{O}-\mathrm{CH}_{2}-\mathrm{CH}_{3}\right)$.

MS (ESI+) $(\mathrm{m} / \mathrm{z})$ : calcd for $\mathrm{C}_{6} \mathrm{H}_{16} \mathrm{NO}_{3}{ }^{+}:[\mathrm{M}+\mathrm{H}]^{+}=134.1176$, found: $m / z=134.1179[\mathrm{M}+\mathrm{H}]^{+}$.

2-(2-(2-Methoxyethoxy)ethoxy)ethylamine $\left(\mathbf{M E}^{3} \mathbf{A}\right)(\mathbf{9})$. Compound $7(20 \mathrm{~g}, 0.13 \mathrm{mmol})$ was allowed to react according to the above procedure, yield: $13 \mathrm{~g}(61 \%)$.

${ }^{1} \mathrm{H}$ NMR $\left(400 \mathrm{MHz}, \mathrm{CDCl}_{3}\right) \delta: 3.52-3.32\left(\mathrm{~m}, 10 \mathrm{H}, \mathrm{NH}_{2}-\mathrm{CH}_{2}-\mathrm{CH}_{2}-\mathrm{O}-\mathrm{CH}_{2}-\mathrm{CH}_{2}-\mathrm{O}-\mathrm{CH}_{2}-\mathrm{CH}_{2}-\mathrm{O}-\mathrm{CH}_{3}\right)$, 3.24 (s, 3H, O-CH3), $2.73\left(\mathrm{~m}, 2 \mathrm{H}, \mathrm{NH}_{2}-\mathrm{CH}_{2}-\mathrm{CH}_{2}-\mathrm{O}\right)$.

${ }^{13} \mathrm{C}$ NMR (100 MHz, $\left.\mathrm{CDCl}_{3}\right) \delta: 73.72\left(1 \mathrm{C}, \mathrm{NH}_{2}-\mathrm{CH}_{2}-\mathrm{CH}_{2}-\mathrm{O}\right), 71.77$ (1C, O- $\left.\mathrm{CH}_{2}-\mathrm{CH}_{2}-\mathrm{O}-\mathrm{CH}_{3}\right), 70.42$ $\left(1 \mathrm{C}, \mathrm{O}-\mathrm{CH}_{2}-\mathrm{CH}_{2}-\mathrm{O}-\mathrm{CH}_{2}-\mathrm{CH}_{2}-\mathrm{O}-\mathrm{CH}_{3}\right), 70.35\left(1 \mathrm{C}, \mathrm{O}-\mathrm{CH}_{2}-\mathrm{CH}_{2}-\mathrm{O}-\mathrm{CH}_{2}-\mathrm{CH}_{2}-\mathrm{O}-\mathrm{CH}_{3}\right), 70.10\left(1 \mathrm{C}, \mathrm{O}-\mathrm{CH}_{2}-\right.$ $\left.\mathrm{CH}_{2}-\mathrm{O}-\mathrm{CH}_{3}\right), 58.82\left(1 \mathrm{C}, \mathrm{O}-\mathrm{CH}_{3}\right), 41.60\left(1 \mathrm{C}, \mathrm{NH}_{2}-\mathrm{CH}_{2}-\mathrm{CH}_{2}-\mathrm{O}\right)$.

MS (ESI+) $(\mathrm{m} / \mathrm{z})$ : calcd for $\mathrm{C}_{7} \mathrm{H}_{18} \mathrm{NO}_{3}{ }^{+}:[\mathrm{M}+\mathrm{H}]^{+}=164.1281$, found: $m / z=164.1285[\mathrm{M}+\mathrm{H}]^{+}$.

\section{Solid-Phase Synthesis of Oligopeptoids}

Oligopeptoid synthesis was carried out on $133 \mathrm{mg}$ of Fmoc-Photolabile Resin SS (0.1 mmol scale, 100200 mesh, 1\% DVB, Advanced ChemTech) using an automated microwave peptide synthesizer (Liberty Blue, CEM Corporation). Three primary amine monomers were synthesized as described above. All other monomers, reagents, and solvents were purchased from commercial sources and directly used without further purification. The oligomer synthetic procedure was adapted from the submonomer approach to solid-phase peptoid synthesis. ${ }^{[4]}$ The resin was deprotected with $20 \%$ 4-methylpiperidine in DMF (v/v) for $30 \mathrm{~s}$ at $75^{\circ} \mathrm{C}$ and then $90 \mathrm{~s}$ at $90^{\circ} \mathrm{C}$, followed by bromoacetylation for $5 \mathrm{~min}$ at $75^{\circ} \mathrm{C}$ with simultaneous addition of $1.5 \mathrm{~mL}$ of $1.0 \mathrm{M}$ bromoacetic acid in DMF and $1.5 \mathrm{~mL}$ of 1.0 M DIC in DMF. Subsequently, halide displacement was performed by addition of $3 \mathrm{~mL}$ of $1.0 \mathrm{M}$ primary amine monomer in NMP followed by an incubation of $5 \mathrm{~min}$ at $75^{\circ} \mathrm{C}$. These bromoacetylation and displacement reactions were alternated until the desired peptoid sequence was achieved. All synthesized peptoids were acetylated by treatment with acetic anhydride to cap the secondary amine end groups.

\section{Photo-cleavage from the resin}

The resin was suspended in $10 \mathrm{~mL}$ DMF in a $20 \mathrm{~mL}$ glass vial and, after purging with nitrogen for $1 \mathrm{~min}$, the vial capped and the suspension stirred while under irradiation at $\sim 25 \mathrm{~mW} \mathrm{~cm}{ }^{-2}$ with $405 \mathrm{~nm}$ for $24 \mathrm{~h}$. The DMF cleavage solution was collected by filtering the suspension through a $0.45 \mu \mathrm{m}$ PTFE syringe 
filter and the resin was rinsed twice with $1 \mathrm{~mL}$ DMF. The filtrate and washings were pooled and evaporated to dryness under vacuum to yield crude peptoid as light yellow oil. 


\section{ESI Mass Spectra of Crude Protected Oligopeptoids}
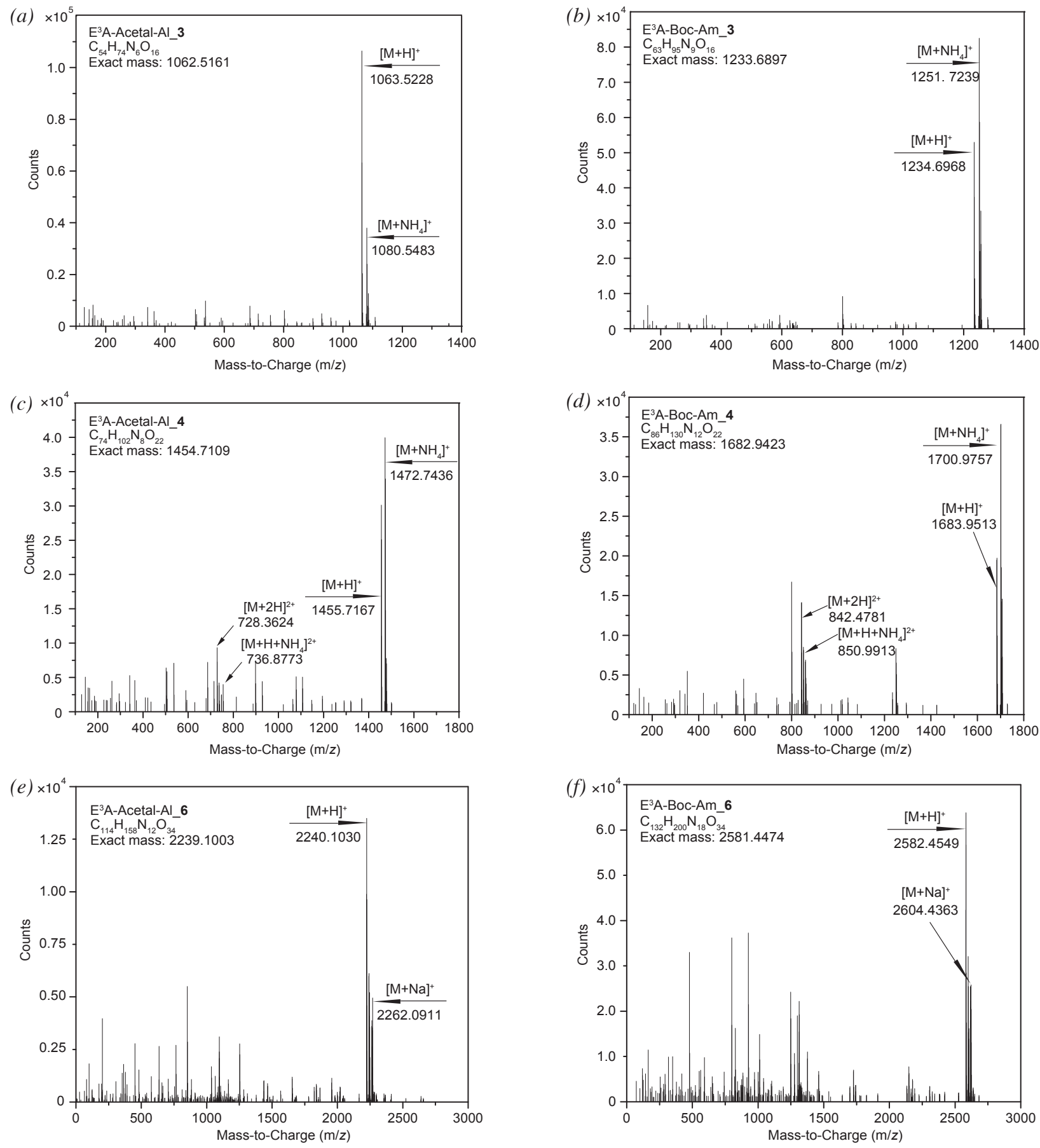

Figure S1. ESI mass spectra of the protected oligopeptoids: trimers $(a) \mathrm{E}^{3} \mathrm{~A}-\mathrm{Acctal}-\mathrm{Al} \_\mathbf{3} \&(b) \mathrm{E}^{3} \mathrm{~A}-\mathrm{B}$ oc-Am_3; tetramers $(c) \mathrm{E}^{3} \mathrm{~A}$-Acetal-Al_4 \& $(d) \mathrm{E}^{3} \mathrm{~A}$-Boc-Am_4; hexamers $(e) \mathrm{E}^{3} \mathrm{~A}$-Acetal-Al_6 $\mathrm{\&}(f) \mathrm{E}^{3} \mathrm{~A}-\mathrm{B}$-Boc-Am_6. All samples were analyzed as crude products after cleavage from the photolabile Fmoc-resin. 


\section{MS (ESI+):}

$\mathrm{E}^{3} \mathrm{~A}$-Acetal-A1_3: calcd for $\mathrm{C}_{54} \mathrm{H}_{74} \mathrm{~N}_{6} \mathrm{O}_{16}:[\mathrm{M}+\mathrm{H}]^{+}=1063.5234,\left[\mathrm{M}+\mathrm{NH}_{4}\right]^{+}=1080.5499$, found: $m / z=$ $1063.5228[\mathrm{M}+\mathrm{H}]^{+}, m / z=1080.5483\left[\mathrm{M}+\mathrm{NH}_{4}\right]^{+}$

$\mathrm{E}^{3} \mathrm{~A}-\mathrm{Boc}-\mathrm{Am} \_$3: calcd for $\mathrm{C}_{63} \mathrm{H}_{95} \mathrm{~N}_{9} \mathrm{O}_{16}:[\mathrm{M}+\mathrm{H}]^{+}=1234.6970,\left[\mathrm{M}+\mathrm{NH}_{4}\right]^{+}=1251.7235$, found: $\mathrm{m} / \mathrm{z}=$ $1234.6968[\mathrm{M}+\mathrm{H}]^{+}, m / z=1251.7239\left[\mathrm{M}+\mathrm{NH}_{4}\right]^{+}$

$\mathrm{E}^{3} \mathrm{~A}$-Acetal-Al_4: calcd for $\mathrm{C}_{74} \mathrm{H}_{102} \mathrm{~N}_{8} \mathrm{O}_{22}:[\mathrm{M}+2 \mathrm{H}]^{2+}=728.3627,\left[\mathrm{M}+\mathrm{H}+\mathrm{NH}_{4}\right]^{2+}=736.8760,[\mathrm{M}+\mathrm{H}]^{+}=$ 1455.7182, $\left[\mathrm{M}+\mathrm{NH}_{4}\right]^{+}=1472.7447$, found: $m / z=728.3624[\mathrm{M}+2 \mathrm{H}]^{2+}, m / z=736.8773\left[\mathrm{M}+\mathrm{H}+\mathrm{NH}_{4}\right]^{2+}$, $m / z=1455.7167[\mathrm{M}+\mathrm{H}]^{+}, m / z=1472.7436\left[\mathrm{M}+\mathrm{NH}_{4}\right]^{+}$

$\mathrm{E}^{3} \mathrm{~A}-\mathrm{Boc}-\mathrm{Am} \_4$ : calcd for $\mathrm{C}_{86} \mathrm{H}_{130} \mathrm{~N}_{12} \mathrm{O}_{22}:[\mathrm{M}+2 \mathrm{H}]^{2+}=842.4784,\left[\mathrm{M}+\mathrm{H}+\mathrm{NH}_{4}\right]^{2+}=850.9917,[\mathrm{M}+\mathrm{H}]^{+}=$ 1683.9496, $\left[\mathrm{M}+\mathrm{NH}_{4}\right]^{+}=1700.9761$, found: $m / z=842.4781[\mathrm{M}+2 \mathrm{H}]^{2+}, m / z=850.9913\left[\left[\mathrm{M}+\mathrm{H}+\mathrm{NH}_{4}\right]^{2+}\right.$, $m / z=1683.9513[\mathrm{M}+\mathrm{H}]^{+}, m / z=1700.9757\left[\mathrm{M}+\mathrm{NH}_{4}\right]^{+}$

$\mathrm{E}^{3}$ A-Acetal-Al_6: calcd for $\mathrm{C}_{114} \mathrm{H}_{158} \mathrm{~N}_{12} \mathrm{O}_{34}:[\mathrm{M}+\mathrm{H}]^{+}=2240.1076,\left[\mathrm{M}+\mathrm{NH}_{4}\right]^{+}=2257.1341,[\mathrm{M}+\mathrm{Na}]^{+}=$ 2262.0895, found: $m / z=2240.1030[\mathrm{M}+\mathrm{H}]^{+}, m / z=2257.1254\left[\mathrm{M}+\mathrm{NH}_{4}\right]^{+}, m / z=2262.0991[\mathrm{M}+\mathrm{Na}]^{+}$ $\mathrm{E}^{3} \mathrm{~A}-\mathrm{Boc}-\mathrm{Am} \_$6: calcd for $\mathrm{C}_{132} \mathrm{H}_{200} \mathrm{~N}_{18} \mathrm{O}_{34}:[\mathrm{M}+\mathrm{H}]^{+}=2582.4547,\left[\mathrm{M}+\mathrm{NH}_{4}\right]^{+}=2599.4812,[\mathrm{M}+\mathrm{Na}]^{+}=$ 2604.4366, found: $m / z=2582.4549[\mathrm{M}+\mathrm{H}]^{+}, m / z=2599.4819\left[\mathrm{M}+\mathrm{NH}_{4}\right]^{+}, m / z=2604.4363[\mathrm{M}+\mathrm{Na}]^{+}$ 
Table S1. Structural information of protected oligopeptoid sequences subjected to ESI mass spectrometry.

\begin{tabular}{|c|c|c|c|}
\hline Code & Structure & Formula & $\begin{array}{c}\text { Exact mass } \\
(\mathrm{g} / \mathrm{mol})\end{array}$ \\
\hline $\mathrm{E}^{3} \mathrm{~A}-\mathrm{Acetal}-\mathrm{Al} \_3$ & & $\mathrm{C}_{54} \mathrm{H}_{74} \mathrm{~N}_{6} \mathrm{O}_{16}$ & 1062.5161 \\
\hline $\mathrm{E}^{3} \mathrm{~A}-\mathrm{Acetal}-\mathrm{Al} \_4$ & & $\mathrm{C}_{74} \mathrm{H}_{102} \mathrm{~N}_{8} \mathrm{O}_{22}$ & 1454.7109 \\
\hline $\mathrm{E}^{3} \mathrm{~A}-\mathrm{Acctal}-\mathrm{Al} \_6$ & & $\mathrm{C}_{114} \mathrm{H}_{158} \mathrm{~N}_{12} \mathrm{O}_{34}$ & 2239.1003 \\
\hline $\mathrm{E}^{3} \mathrm{~A}-\mathrm{Boc}-\mathrm{Am} \_3$ & & $\mathrm{C}_{63} \mathrm{H}_{95} \mathrm{~N}_{9} \mathrm{O}_{16}$ & 1233.6897 \\
\hline $\mathrm{E}^{3} \mathrm{~A}-\mathrm{Boc}-\mathrm{Am} \_4$ & & $\mathrm{C}_{86} \mathrm{H}_{130} \mathrm{~N}_{12} \mathrm{O}_{22}$ & 1682.9423 \\
\hline $\mathrm{E}^{3} \mathrm{~A}-\mathrm{Boc}-\mathrm{Am} \_6$ & & $\mathrm{C}_{132} \mathrm{H}_{200} \mathrm{~N}_{18} \mathrm{O}_{34}$ & 2581.4474 \\
\hline
\end{tabular}




\section{Deprotection of Acetal-Protected Oligopeptoids}

The acetal-protected crude peptoid products were treated with $3 \mathrm{~mL} \mathrm{40 \%} \mathrm{TFA} \mathrm{in} \mathrm{DI} \mathrm{water} \mathrm{for} 25$ min to yield the aldehyde-functionalized peptoids $\mathrm{E}^{3} \mathrm{~A}-\mathrm{Al} \_3, \mathrm{E}^{3} \mathrm{~A}-\mathrm{Al} \_$4, and $\mathrm{E}^{3} \mathrm{~A}-\mathrm{Al} \_$. The reaction mixtures were concentrated and further dried under high vacuum. The crude products were then reconstituted in $20 \% \sim 30 \%$ HPLC grade $\mathrm{MeCN} / \mathrm{H}_{2} \mathrm{O}(\mathrm{v} / \mathrm{v})$ and purified by preparative RP-HPLC.

\section{Deprotection of Boc-Protected Oligopeptoids}

The Boc-protected peptoid crude products were treated with $3 \mathrm{~mL} \mathrm{20 \%} \mathrm{TFA} \mathrm{in} \mathrm{DCM} \mathrm{for} 10 \sim 17.5$ min to

yield the amine-functionalized peptoids $\mathrm{E}^{3} \mathrm{~A}-\mathrm{Am} \_3, \mathrm{E}^{3} \mathrm{~A}-\mathrm{Am} \_4$, and $\mathrm{E}^{3} \mathrm{~A}-\mathrm{Am} \_$6. The reaction mixtures were evaporated by blowing with a gentle stream of nitrogen and further dried by high vacuum. The crude products were dissolved in $\mathrm{MeCN} / \mathrm{H}_{2} \mathrm{O}$ and subjected to $1 \mathrm{M} \mathrm{NaHCO}_{3}$ to adjust the $\mathrm{pH}$ to $\sim 7.6$, followed by evaporated to dryness under vacuum. The crude products were then re-dissolved in $\mathrm{MeCN}$ and filtered through a syringe filter with a $0.45 \mu \mathrm{m}$ PTFE membrane. The filtrates were combined and evaporated to dryness to yield yellow oil. The crude oil was reconstituted in $20 \% \sim 30 \% \mathrm{HPLC}$ grade $\mathrm{MeCN} / \mathrm{H}_{2} \mathrm{O}(\mathrm{v} / \mathrm{v}$ ) and purified by preparative RP-HPLC.

\section{Preparative RP-HPLC Method}

All deprotected peptoids were purified by preparative HPLC using a linear gradient of $\mathrm{H}_{2} \mathrm{O}$ (A) and $\mathrm{MeCN}$ (B) at a flow rate of $12 \mathrm{~mL} / \mathrm{min}$.

Prep HPLC method for E ${ }^{3} \mathrm{~A}-\mathrm{Am} \_3, \mathrm{E}^{3} \mathrm{~A}-\mathrm{Am} \_4$, and $\mathrm{E}^{3} \mathrm{~A}-\mathrm{Am} \_6$ : (1) $20 \% \mathrm{~B}, 0.1-4.1 \mathrm{~min}$; (2) 20\% $65 \% \mathrm{~B}, 4.1-30.1 \mathrm{~min}$; (3) $65 \%-20 \% \mathrm{~B}, 30.1-32.1 \mathrm{~min}$.

Prep HPLC method for E ${ }^{3} \mathrm{~A}-\mathrm{Al} \_3$ and $\mathrm{E}^{3} \mathrm{~A}-\mathrm{Al} \_4$ : (1) 30\% B, $0.1-4.1 \mathrm{~min}$; (2) 30\% - 70\% B, $4.1-19.1$ $\min$; (3) 70\% - 30\% B, $19.1-21.1 \mathrm{~min}$.

Prep HPLC method for E ${ }^{3} \mathrm{~A}-\mathrm{Al} \_6$ : (1) 30\% B, 0.1 - $4.1 \mathrm{~min}$; (2) $30 \%-70 \% \mathrm{~B}, 4.1-19.1 \mathrm{~min}$; (3) 70\% B, 19.1 - $22.1 \mathrm{~min}$; (4) 70\% - 30\% B, $22.1-24.1 \mathrm{~min}$.

The purified fractions were combined, concentrated, reconstituted in $50 \% \mathrm{MeCN} / \mathrm{H}_{2} \mathrm{O}(\mathrm{v} / \mathrm{v})$, frozen with liquid nitrogen and lyophilized to afford fluffy white powders. The purity of the collected, aldehyde- and amine-functionalized peptoids was examined by analytical RP-HPLC with a linear gradient of $\mathrm{MeCN} / \mathrm{H}_{2} \mathrm{O}$ from $30 \%$ to $70 \%$. 
ESI Mass Spectra of Purified, Aldehyde- and Amine-Functionalized Oligopeptoids
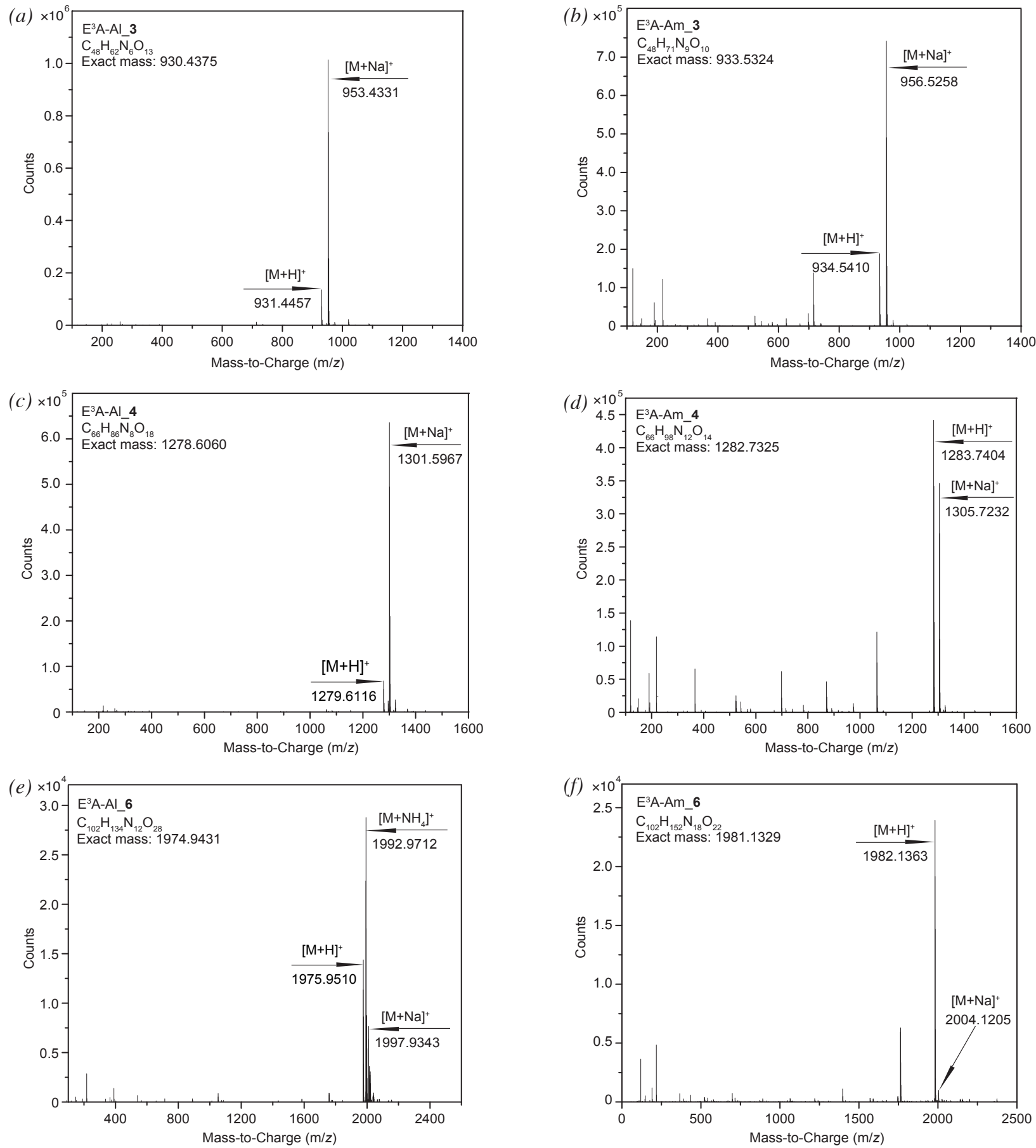

Figure S2. ESI mass spectra of aldehyde- and amine-functionalized oligopeptoids purified by preparative RP-HPLC: trimers $(a) \mathrm{E}^{3} \mathrm{~A}-\mathrm{Al} \_3$ \& $(b) \mathrm{E}^{3} \mathrm{~A}-\mathrm{Am} \_3$; tetramers $(c) \mathrm{E}^{3} \mathrm{~A}-\mathrm{Al} \_4$ \& $(d) \mathrm{E}^{3} \mathrm{~A}-\mathrm{Am} \_4$; hexamers $(e) \mathrm{E}^{3} \mathrm{~A}-\mathrm{Al} \_6$ \& $(f)$ E ${ }^{3} \mathrm{~A}-\mathrm{Am} 6$. 


\section{MS (ESI+):}

$\mathrm{E}^{3} \mathrm{~A}-\mathrm{Al} \_$3: calcd for $\mathrm{C}_{48} \mathrm{H}_{62} \mathrm{~N}_{6} \mathrm{O}_{13}:[\mathrm{M}+\mathrm{H}]^{+}=931.4448,[\mathrm{M}+\mathrm{Na}]^{+}=953.4267$, found: $\mathrm{m} / z=931.4457$ $[\mathrm{M}+\mathrm{H}]^{+}, m / z=953.4331[\mathrm{M}+\mathrm{Na}]^{+}$

$\mathrm{E}^{3} \mathrm{~A}-\mathrm{Am} \_3$ : calcd for $\mathrm{C}_{48} \mathrm{H}_{71} \mathrm{~N}_{9} \mathrm{O}_{10}:[\mathrm{M}+\mathrm{H}]^{+}=934.5397,[\mathrm{M}+\mathrm{Na}]^{+}=956.5216$, found: $\mathrm{m} / z=934.5410$ $[\mathrm{M}+\mathrm{H}]^{+}, m / z=956.5258[\mathrm{M}+\mathrm{Na}]^{+}$

$\mathrm{E}^{3} \mathrm{~A}-\mathrm{Al}$ 4: calcd for $\mathrm{C}_{66} \mathrm{H}_{86} \mathrm{~N}_{8} \mathrm{O}_{18}:[\mathrm{M}+\mathrm{H}]^{+}=1279.6133,[\mathrm{M}+\mathrm{Na}]^{+}=1301.5952$, found: $\mathrm{m} / z=1279.6116$ $[\mathrm{M}+\mathrm{H}]^{+}, m / z=1301.5967[\mathrm{M}+\mathrm{Na}]^{+}$

$\mathrm{E}^{3} \mathrm{~A}-\mathrm{Am}$ 4: calcd for $\mathrm{C}_{66} \mathrm{H}_{98} \mathrm{~N}_{12} \mathrm{O}_{14}:[\mathrm{M}+\mathrm{H}]^{+}=1283.7398,[\mathrm{M}+\mathrm{Na}]^{+}=1305.7217$, found: $m / z=1283.7404$ $[\mathrm{M}+\mathrm{H}]^{+}, m / z=1305.7232[\mathrm{M}+\mathrm{Na}]^{+}$

E 3 A-Al_6: calcd for $\mathrm{C}_{102} \mathrm{H}_{134} \mathrm{~N}_{12} \mathrm{O}_{28}:[\mathrm{M}+\mathrm{H}]^{+}=1975.9504,\left[\mathrm{M}+\mathrm{NH}_{4}\right]^{+}=1992.9769,[\mathrm{M}+\mathrm{Na}]^{+}=$ 1997.9323, found: $m / z=1975.9510[\mathrm{M}+\mathrm{H}]^{+}, m / z=1992.9712\left[\mathrm{M}+\mathrm{NH}_{4}\right]^{+}, m / z=1997.9343[\mathrm{M}+\mathrm{Na}]^{+}$ $\mathrm{E}^{3} \mathrm{~A}-\mathrm{Am}$ 6: calcd for $\mathrm{C}_{102} \mathrm{H}_{152} \mathrm{~N}_{18} \mathrm{O}_{22}:[\mathrm{M}+\mathrm{H}]^{+}=1982.1402,[\mathrm{M}+\mathrm{Na}]^{+}=2004.1221$, found: $\mathrm{m} / z=$ $1982.1363[\mathrm{M}+\mathrm{H}]^{+}, m / z=2004.1205[\mathrm{M}+\mathrm{Na}]^{+}$

\section{ESI Mass Spectra of Purified, Aldehyde- and Amine-Functionalized Oligopeptoids} Incorporating $\mathrm{ME}^{3} \mathrm{~A}$ Spacer
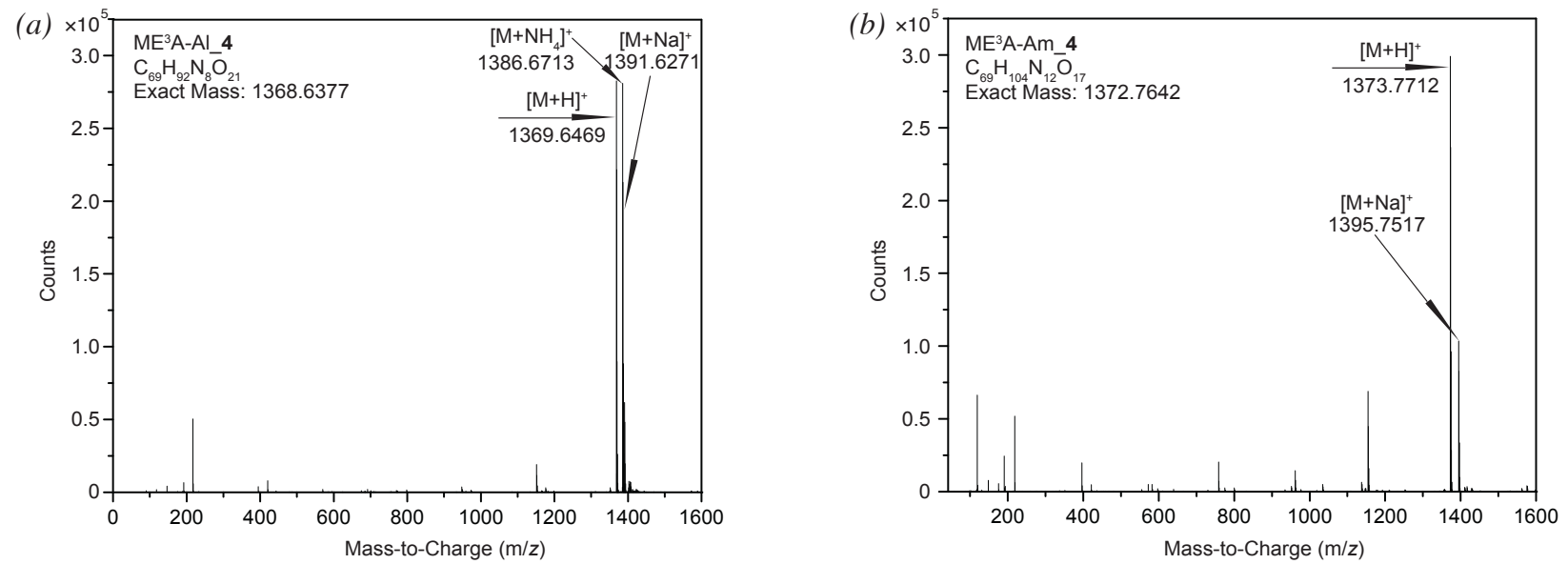

Figure S3. ESI mass spectra of aldehyde- and amine-functionalized oligopeptoids incorporating $\mathrm{ME}^{3} \mathrm{~A}$ spacer purified by preparative RP-HPLC: tetramers $(a) \mathrm{ME}^{3} \mathrm{~A}-\mathrm{Al} \_4$ \& $(b) \mathrm{ME}^{3} \mathrm{~A}-\mathrm{Am} \_4$.

ME ${ }^{3}$ A-Al_4: calcd for $\mathrm{C}_{69} \mathrm{H}_{92} \mathrm{~N}_{8} \mathrm{O}_{21}:[\mathrm{M}+\mathrm{H}]^{+}=1369.6450,\left[\mathrm{M}+\mathrm{NH}_{4}\right]^{+}=1386.6716,[\mathrm{M}+\mathrm{Na}]^{+}=$ 1391.6270, found: $m / z=1369.6469[\mathrm{M}+\mathrm{H}]^{+}, m / z=\left[\mathrm{M}+\mathrm{NH}_{4}\right]^{+}=1386.6713, m / z=1391.6271[\mathrm{M}+\mathrm{Na}]^{+}$ ME ${ }^{3} \mathrm{~A}-\mathrm{Am} \_$4: calcd for $\mathrm{C}_{69} \mathrm{H}_{104} \mathrm{~N}_{12} \mathrm{O}_{17}:[\mathrm{M}+\mathrm{H}]^{+}=1373.7715,[\mathrm{M}+\mathrm{Na}]^{+}=1395.7534$, found: $\mathrm{m} / z=$ $1373.7712[\mathrm{M}+\mathrm{H}]^{+}, m / z=1395.7517[\mathrm{M}+\mathrm{Na}]^{+}$ 


\section{NMR Spectra of Purified, Aldehyde- and Amine-Functionalized Oligopeptoids}

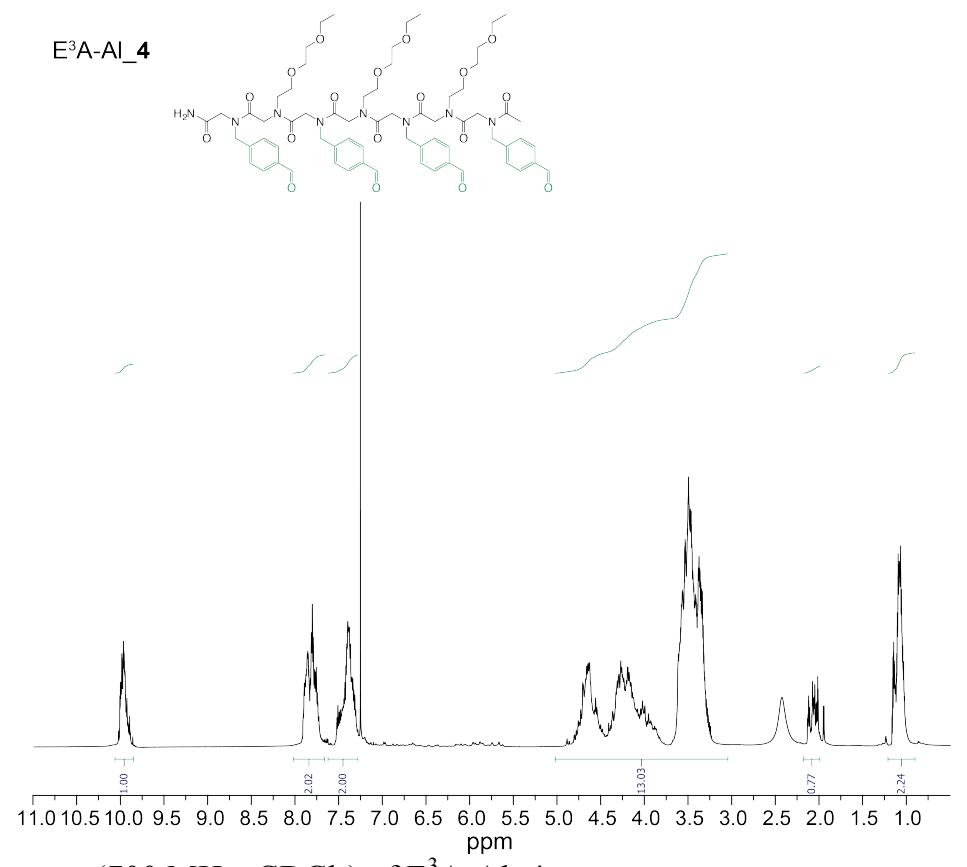

Figure S4. ${ }^{1} \mathrm{H}$ NMR spectrum (700 MHz, $\left.\mathrm{CDCl}_{3}\right)$ of $\mathrm{E}^{3} \mathrm{~A}-\mathrm{Al} \_4$.

$\mathrm{E}^{3} \mathrm{~A}-\mathrm{Al} \mathbf{3}$

${ }^{1} \mathrm{H}$ NMR (700 MHz, $\mathrm{CDCl}_{3}$ ) $\delta$ : 9.96-10.01 (m, Ar-CHO), 7.72-7.92 (m, Ar), 7.36-7.48 (m, Ar), 4.644.87 (m, Ar- $\mathrm{CH}_{2}-\mathrm{N}-$ ), 3.88-4.34 (m, -N-CH$-\mathrm{CH}_{2}-\mathrm{O}-,-\mathrm{N}-\mathrm{CH}_{2}-\mathrm{CO}-$ ), 3.34-3.63 (m, -O-CH$-\mathrm{CH}_{2}-\mathrm{O}-$, -O$\left.\mathrm{CH}_{2}-\mathrm{CH}_{3},-\mathrm{N}-\mathrm{CH}_{2}-\mathrm{CH}_{2}-\mathrm{O}-\right), 2.06-2.18\left(\mathrm{~m},-\mathrm{CO}-\mathrm{CH}_{3}\right), 1.05-1.17\left(\mathrm{~m},-\mathrm{O}-\mathrm{CH}_{2}-\mathrm{CH}_{3}\right)$

$\mathrm{E}^{3} \mathrm{~A}-\mathrm{Al} 4$

${ }^{1} \mathrm{H}$ NMR $\bar{R}\left(700 \mathrm{MHz}, \mathrm{CDCl}_{3}\right) \delta$ : 9.95-10.01 (m, Ar-CHO), 7.75-7.90 (m, Ar), 7.35-7.51 (m, Ar), 4.56-

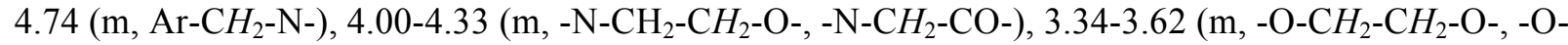
$\left.\mathrm{CH}_{2}-\mathrm{CH}_{3},-\mathrm{N}-\mathrm{CH}_{2}-\mathrm{CH}_{2}-\mathrm{O}-\right), 1.95-2.12\left(\mathrm{~m},-\mathrm{CO}-\mathrm{CH}_{3}\right), 1.04-1.16\left(\mathrm{~m},-\mathrm{O}-\mathrm{CH}_{2}-\mathrm{CH}_{3}\right)$

$\mathrm{E}^{3} \mathrm{~A}-\mathrm{Al} 6$

${ }^{1} \mathrm{H}$ NMR $\left(700 \mathrm{MHz}, \mathrm{CDCl}_{3}\right.$ ) $\delta$ : 9.95-10.00 (br, Ar-CHO), 7.72-7.89 (br, Ar), 7.33-7.49 (br, Ar), 4.35-

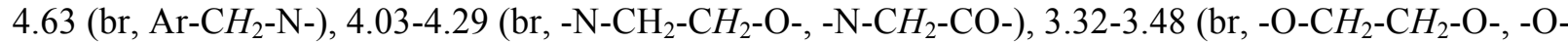
$\mathrm{CH}_{2}-\mathrm{CH}_{3},-\mathrm{N}-\mathrm{CH}_{2}-\mathrm{CH}_{2}-\mathrm{O}-$ ), 2.00-2.12 (br, $-\mathrm{CO}-\mathrm{CH}_{3}$ ), 1.05-1.10 (br, $-\mathrm{O}-\mathrm{CH}_{2}-\mathrm{CH}_{3}$ ) 


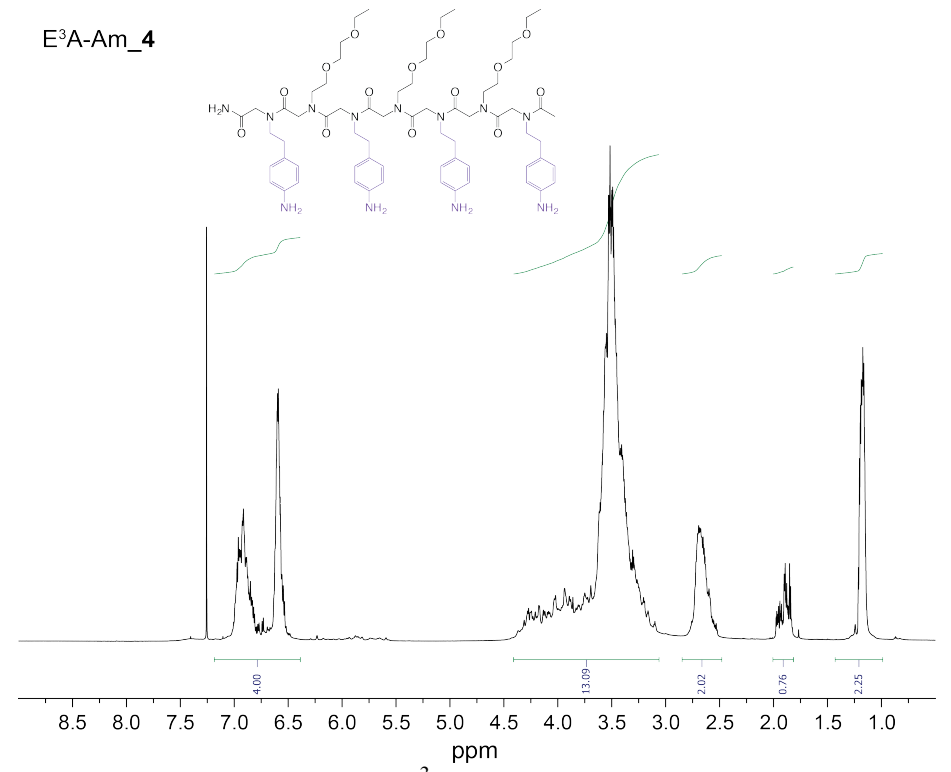

Figure S5. ${ }^{1} \mathrm{H}$ NMR spectrum $\left(700 \mathrm{MHz}, \mathrm{CDCl}_{3}\right)$ of $\mathrm{E}^{3} \mathrm{~A}-\mathrm{Am} \_4$.

$\mathrm{E}^{3} \mathrm{~A}-\mathrm{Am} \_3$

${ }^{1} \mathrm{H}$ NMR (700 MHz, $\mathrm{CDCl}_{3}$ ) $\delta:$ 6.78-6.94 (m, Ar), 6.56-6.60 (m, Ar), 3.73-4.27 (m, -N-CH $-\mathrm{CO}-,-\mathrm{N}-$

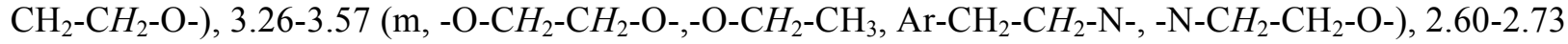

(m, $\left.-\mathrm{Ar}-\mathrm{CH}_{2}-\mathrm{CH}_{2}-\mathrm{N}-\right), 1.80-1.97$ (m, $\left.-\mathrm{CO}-\mathrm{CH}_{3}\right), 1.17-1.21\left(\mathrm{~m},-\mathrm{O}-\mathrm{CH}_{2}-\mathrm{CH}_{3}\right)$

$\mathrm{E}^{3} \mathrm{~A}-\mathrm{Am} 4$

${ }^{1} \mathrm{H}$ NMR $\left(700 \mathrm{MHz}, \mathrm{CDCl}_{3}\right) \delta$ : 6.83-6.97 (m, Ar), 6.54-6.60 (m, Ar), 3.75-4.31 (m, -N-CH $\mathrm{CH}_{2} \mathrm{CO}-,-\mathrm{N}-$

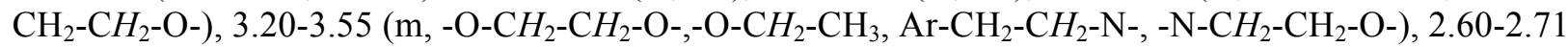

(m, $\left.-\mathrm{Ar}-\mathrm{CH}_{2}-\mathrm{CH}_{2}-\mathrm{N}-\right)$, 1.85-1.98 (m, $\left.-\mathrm{CO}-\mathrm{CH}_{3}\right), 1.17-1.20$ (m, $-\mathrm{O}-\mathrm{CH}_{2}-\mathrm{CH}_{3}$ )

$\mathrm{E}^{3} \mathrm{~A}-\mathrm{Am} 6$

${ }^{1} \mathrm{H}$ NMR (700 MHz, $\mathrm{CDCl}_{3}$ ) $\delta$ : 6.91-6.98 (br, Ar), 6.60 (br, Ar), 3.89-4.37 (br, -N-CH $\mathrm{CH}_{2} \mathrm{CO}-$, , N-CH$\mathrm{CH}_{2}-\mathrm{O}-$ ), 3.22-3.60 (br, -O- $\mathrm{CH}_{2}-\mathrm{CH}_{2}-\mathrm{O}-,-\mathrm{O}-\mathrm{CH}_{2}-\mathrm{CH}_{3}, \mathrm{Ar}_{-} \mathrm{CH}_{2}-\mathrm{CH}_{2}-\mathrm{N}-,-\mathrm{N}-\mathrm{CH}_{2}-\mathrm{CH}_{2}-\mathrm{O}-$ ), 2.67-2.70 (br, - $\mathrm{Ar}-\mathrm{CH}_{2}-\mathrm{CH}_{2}-\mathrm{N}-$ ), 1.87-1.99 (br, $-\mathrm{CO}-\mathrm{CH}_{3}$ ), 1.17-1.20 (br, $-\mathrm{O}-\mathrm{CH}_{2}-\mathrm{CH}_{3}$ ) 
NMR Spectra of Purified, Aldehyde- and Amine-Functionalized Oligopeptoids Incorporating $\mathrm{ME}^{3} \mathrm{~A}$ Spacer

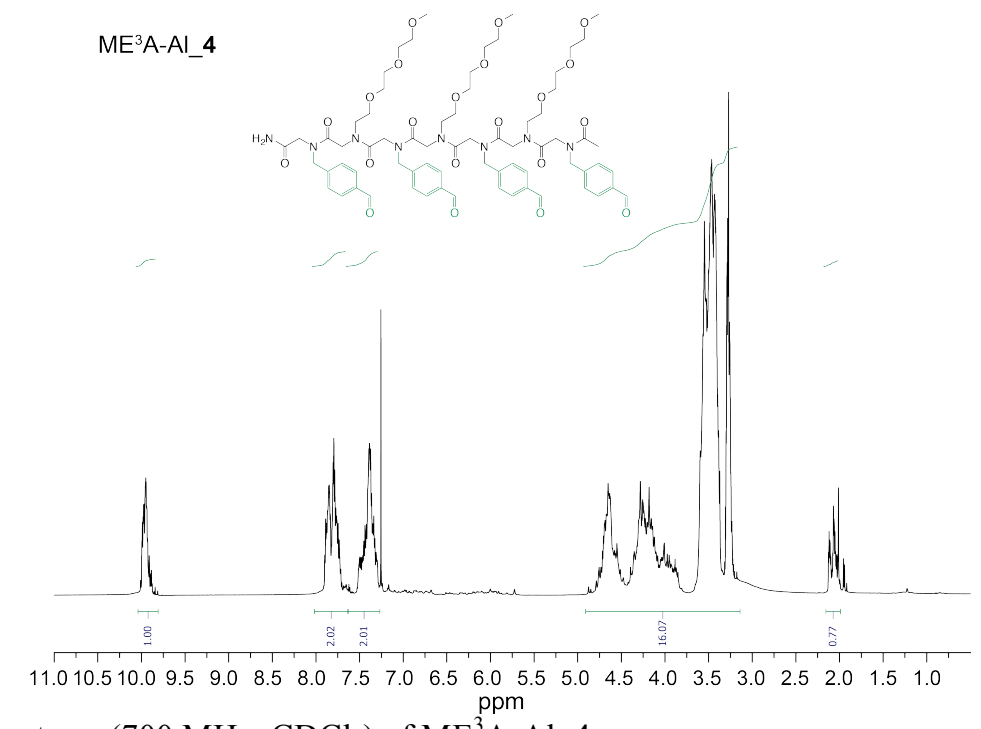

Figure S6. ${ }^{~}{ }^{\mathrm{H}} \mathrm{NMR}$ spectrum $\left(700 \mathrm{MHz}, \mathrm{CDCl}_{3}\right)$ of $\mathrm{ME}^{3} \mathrm{Am}-\mathrm{Al} \_4$.

$\mathrm{ME}^{3} \mathrm{~A}-\mathrm{Al} \_4$

${ }^{1} \mathrm{H}$ NMR (700 MHz, $\mathrm{CDCl}_{3}$ ) $8:$ 9.89-9.99 (m, Ar-CHO), 7.75-7.89 (m, Ar), 7.31-7.50 (m, Ar), 4.56-4.76

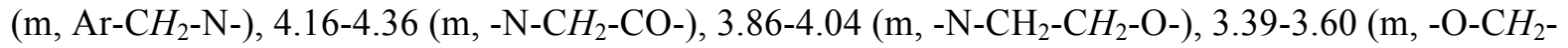
$\left.\mathrm{CH}_{2}-\mathrm{O}-\right)$, 3.26-3.30 (m, -O-CH $\left.3,-\mathrm{N}-\mathrm{CH}_{2}-\mathrm{CH}_{2}-\mathrm{O}-\right)$, 2.02-2.12 (m, - $\mathrm{CO}-\mathrm{CH}_{3}$ ).

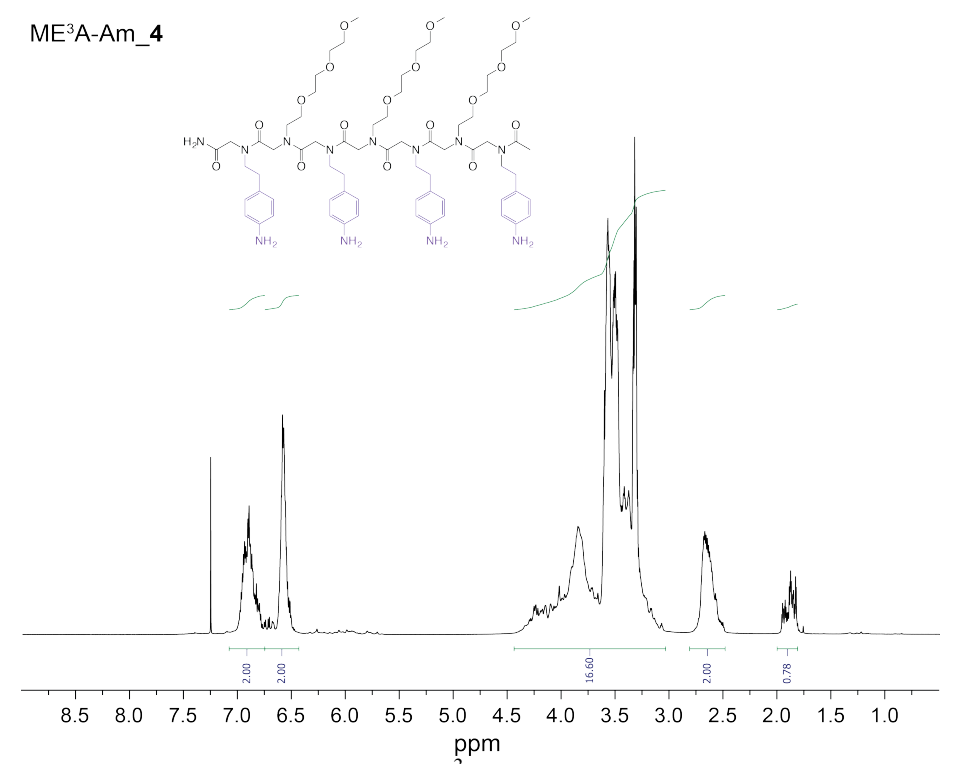

Figure S7. ${ }^{1} \mathrm{H}$ NMR spectrum (700 MHz, $\mathrm{CDCl}_{3}$ ) of $\mathrm{ME}^{3} \mathrm{~A}-\mathrm{Am} \_4$.

$\mathrm{ME}^{3} \mathrm{~A}-\mathrm{Am} \mathbf{4}$

${ }^{1} \mathrm{H}$ NMR (700 MHz, $\mathrm{CDCl}_{3}$ ) $\delta:$ 6.84-6.96 (m, Ar), 6.55-6.59 (m, Ar), 3.73-4.31 (br, -N-CH $\left.\mathrm{H}_{2}-\mathrm{CO}-\right), 3.49-$ 3.61 (m, -O-CH $\mathrm{CH}_{2}-\mathrm{CH}_{2}-\mathrm{O}-, \mathrm{Ar}^{-} \mathrm{CH}_{2}-\mathrm{CH}_{2}-\mathrm{N}-,-\mathrm{N}-\mathrm{CH}_{2}-\mathrm{CH}_{2}-\mathrm{O}-$ ), 3.32-3.34 (m, -O-CH3,$-\mathrm{N}-\mathrm{CH}_{2}-\mathrm{CH}_{2}-\mathrm{O}-$ ), 2.58-2.69 (br, $\left.\mathrm{Ar}-\mathrm{CH}_{2}-\mathrm{CH}_{2}-\mathrm{N}-\right)$, 1.84-1.96 (m, $\left.-\mathrm{CO}-\mathrm{CH}_{3}\right)$. 


\section{Analytical HPLC Traces of Purified, Aldehyde- and Amine-Functionalized Oligopeptoids}
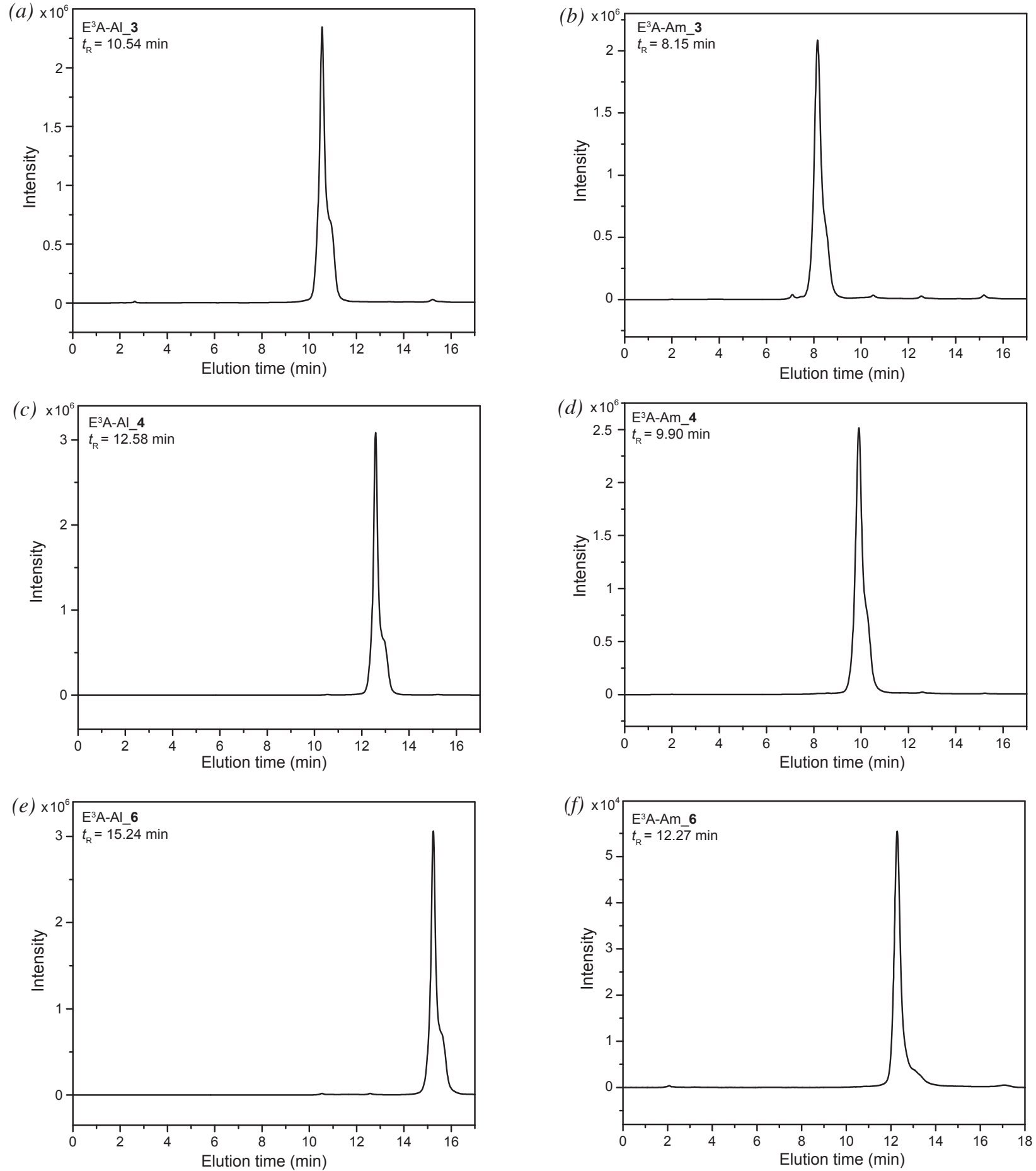

Figure S8. Analytical HPLC traces of aldehyde- and amine-functionalized oligopeptoids: trimers $(a) \mathrm{E}^{3} \mathrm{~A}-\mathrm{Al} 3$ (purity 98\%) \& (b) $\mathrm{E}^{3} \mathrm{~A}-\mathrm{Am} \_3$ (purity 98\%); tetramers (c) $\mathrm{E}^{3} \mathrm{~A}-\mathrm{Al} \_4$ (purity 99\%) \& (d) $\mathrm{E}^{3} \mathrm{~A}-\mathrm{Am} \_4$ (purity $98 \%$ ); hexamers (e) $\mathrm{E}^{3} \mathrm{~A}-\mathrm{Al} 6$ (purity 98\%) \& $(f) \mathrm{E}^{3} \mathrm{~A}-\mathrm{Am} 6$ (purity $99 \%$ ). Analytical HPLC method: flow rate at 1 $\mathrm{mL} / \mathrm{min} ; \mathrm{A}: \mathrm{H}_{2} \mathrm{O}$, B: $\mathrm{MeCN} ; 30 \%-70 \%$ B $0-15 \mathrm{~min}, 70 \%-30 \%$ B $15-18 \mathrm{~min}$. 


\section{Analytical HPLC Traces of Purified, Aldehyde- and Amine-Functionalized Oligopeptoids Incorporating $\mathrm{ME}^{3} \mathrm{~A}$ Spacer}
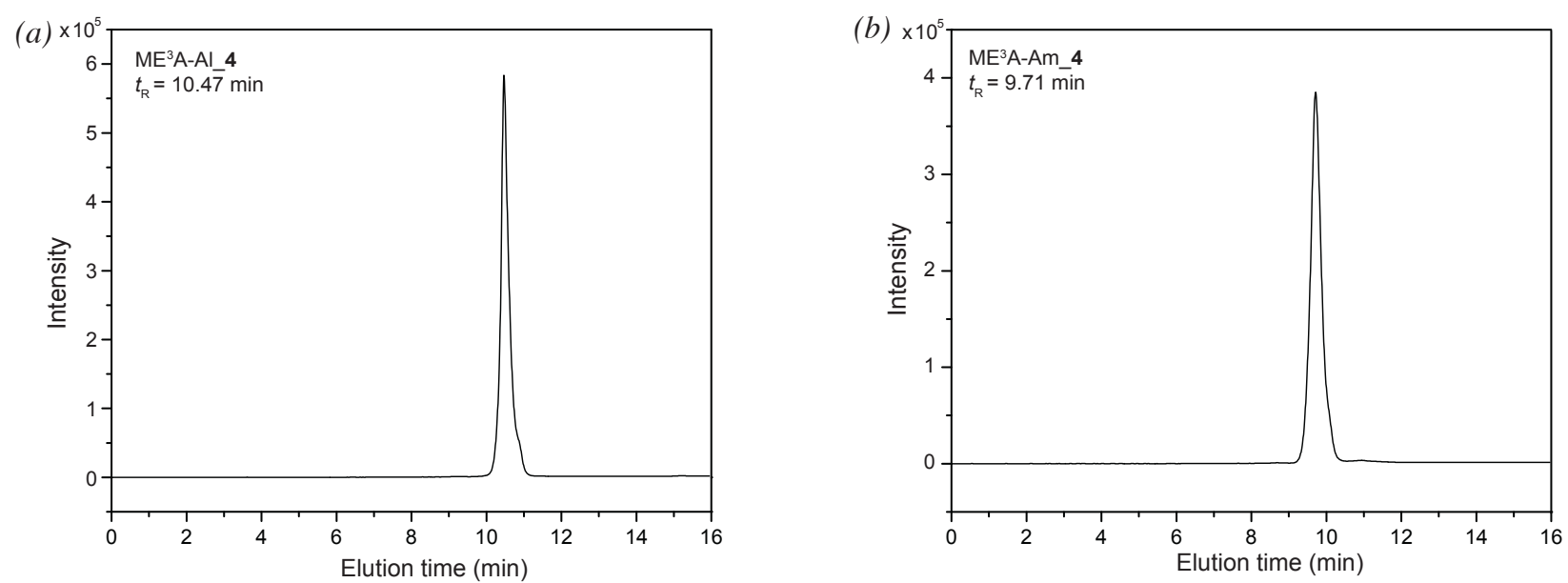

Figure S9. Analytical HPLC traces of aldehyde- and amine-functionalized oligopeptoids incorporating $\mathrm{ME}^{3} \mathrm{~A}$ spacer: tetramers (a) $\mathrm{ME}^{3} \mathrm{~A}-\mathrm{Al} \_4$ (purity 98\%) \& (b) $\mathrm{ME}^{3} \mathrm{~A}-\mathrm{Am} \_4$ (purity 97\%). Analytical HPLC method: flow rate at $1 \mathrm{~mL} / \mathrm{min} ; \mathrm{A}: \mathrm{H}_{2} \mathrm{O}, \mathrm{B}: \mathrm{MeCN} ; 20 \%-80 \%$ B $0-13 \mathrm{~min}, 80 \%-20 \%$ B $13-16 \mathrm{~min}$.

\section{Analytical GPC Traces of Purified, Aldehyde- and Amine-Functionalized Oligopeptoids}
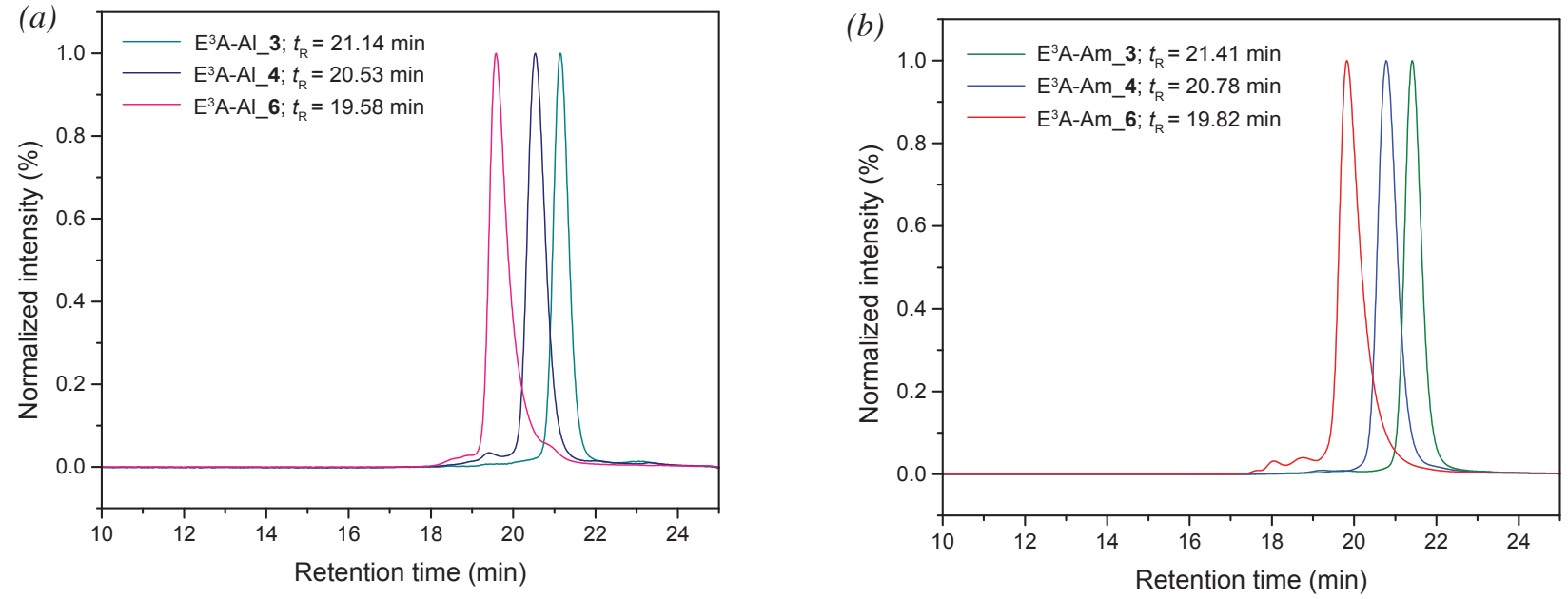

Figure S10. Analytical GPC of aldehyde- and amine-functionalized oligopeptoids: (a) aldehyde peptoid trimer, tetramer, and hexamer; $(b)$ amine peptoid trimer, tetramer, and hexamer. Analytical GPC method: isocratic flow rate at $1 \mathrm{~mL} / \mathrm{min}$; eluent: $\mathrm{CHCl}_{3} / \mathrm{CH}_{3} \mathrm{OH} / \mathrm{Et}_{3} \mathrm{~N}(94 / 4 / 2, \mathrm{v} / \mathrm{v} / \mathrm{v})$. 
Table S2. Structural information of aldehyde- and amine-functionalized oligopeptoid sequences subjected to ESI mass spectrometry, HPLC and GPC analysis.

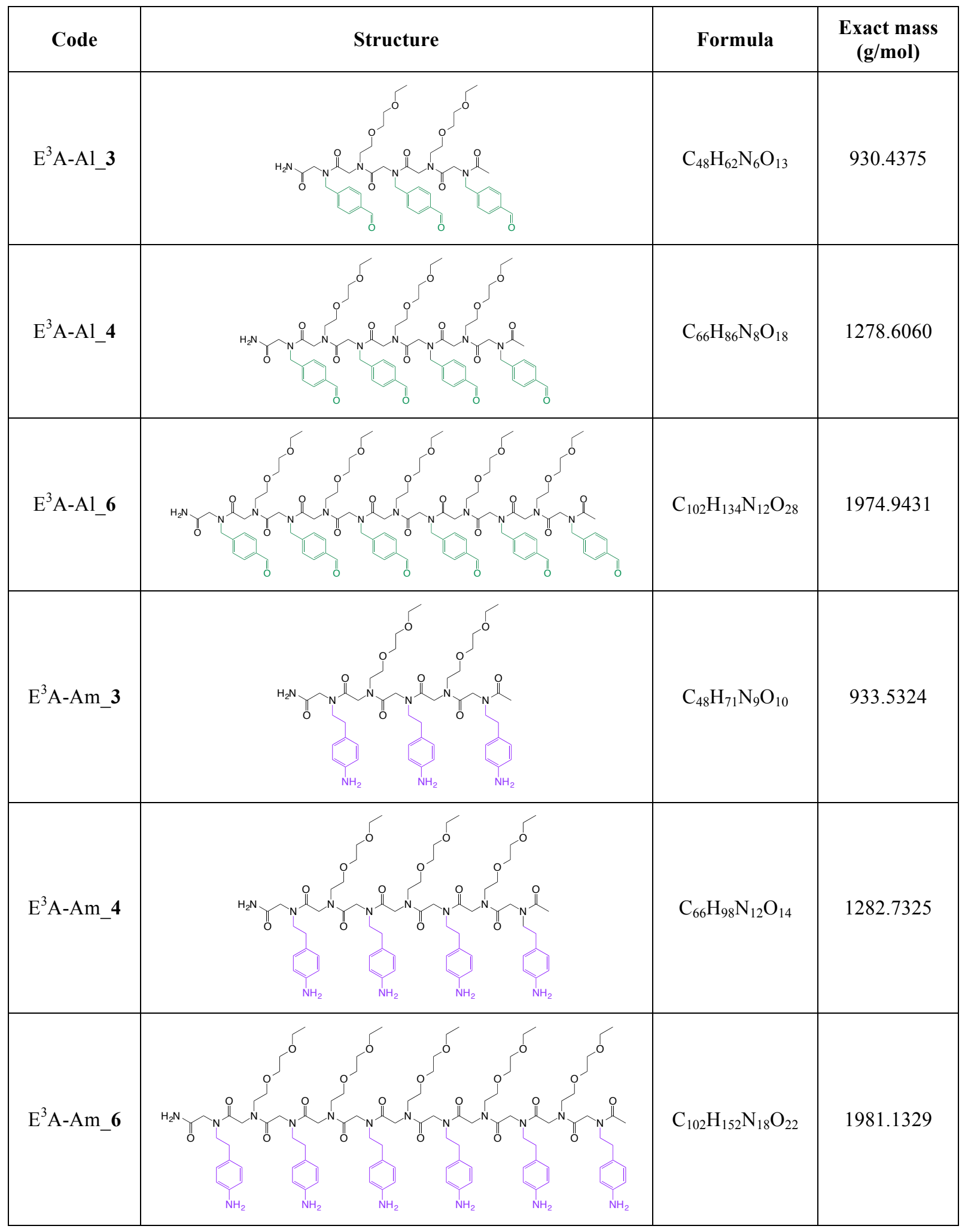




\section{General Procedure for Self-Assembly of Molecular Ladders of Length $n$}

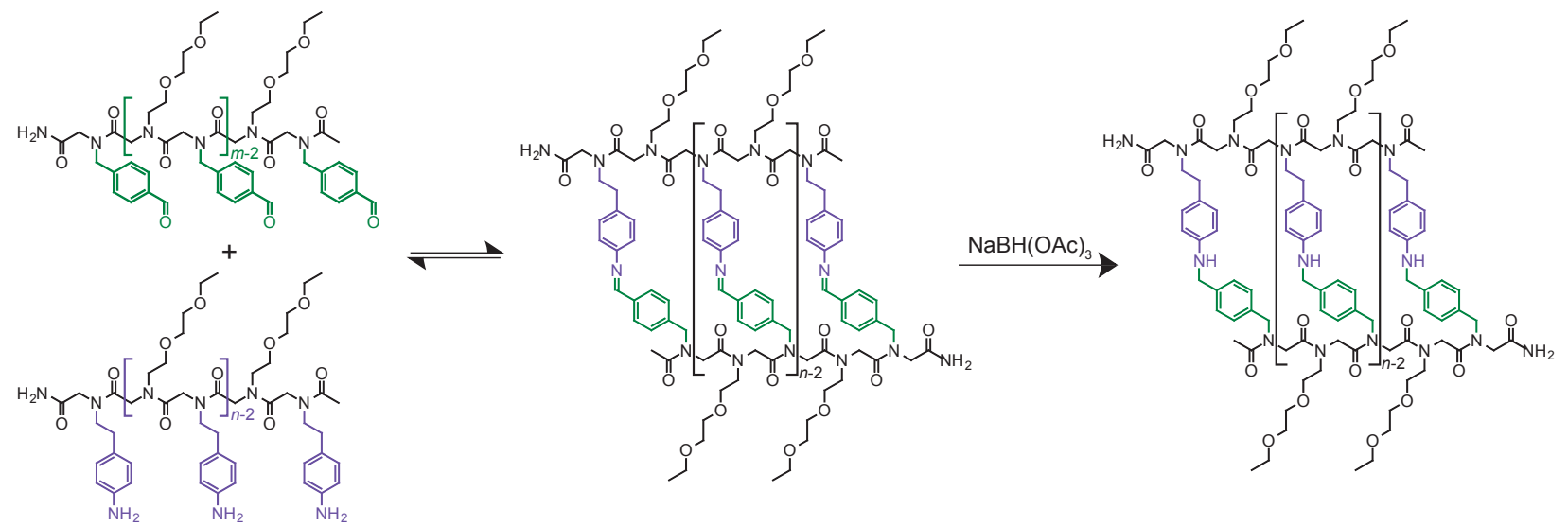

Scheme S4. Dimerization of peptoid-based molecular ladders via imine metathesis $(\boldsymbol{m}=\boldsymbol{n})$.

The method to dimerize aldehyde- and amine-functionalized peptoid oligomers was adapted from a published approach. ${ }^{[5]} 1 \mu \mathrm{mol}$ of the peptoid oligomer $\mathrm{E}^{3} \mathrm{~A}-\mathrm{Al} \boldsymbol{m}$ was mixed with $1 \mu \mathrm{mol}$ of its complementary strand $\mathrm{E}^{3} \mathrm{~A}-\mathrm{Am} \_\boldsymbol{n}$, where $\boldsymbol{m}=\boldsymbol{n}$, in $2 \mathrm{~mL} \mathrm{CHCl}_{3}$. To the reaction mixture, $\mathrm{Sc}(\mathrm{OTf})_{3}(0.04$ eq. per imine bond, $10 \mathrm{mM}$ stock solution dissolved in $\mathrm{MeCN}$ ) was added and the mixture allowed to stir overnight. The reaction mixture was then directly analyzed by MALDI mass spectrometry and analytical GPC. Sodium triacetoxyborohydride $\left(\mathrm{NaBH}(\mathrm{OAc})_{3}\right)$ was subsequently added (10 equivalents per imine bond), the reaction mixture was stirred overnight to reduce the inter-strand imine groups to secondary amines, and the mixture was characterized by MALDI mass spectrometry.

\section{General Procedure for Vernier-Templated Self-Assembly of Molecular Ladders of Length} $m \times n$

A $1 \mu \mathrm{mol}$ self-assembly scale was performed by mixing $\mathrm{E}^{3} \mathrm{~A}-\mathrm{A} \_\boldsymbol{m}$ with its complementary strand $\mathrm{E}^{3} \mathrm{~A}$ Am_n, where $\boldsymbol{m} \neq \boldsymbol{n}$ but in a 1:1 aldehyde:amine stoichiometric ratio, in $2 \mathrm{~mL} \mathrm{CHCl}_{3}$. To the reaction mixture, $\mathrm{Sc}(\mathrm{OTf})_{3}(0.04$ eq. per imine bond, $10 \mathrm{mM}$ stock solution dissolved in $\mathrm{MeCN})$ was added and the mixture allowed to stir for 7 days. The reaction mixture was again directly characterized by MALDI mass spectrometry and analytical GPC, after which $\mathrm{NaBH}(\mathrm{OAc})_{3}$ was added to reduce the inter-strand imine groups and the mixture was again characterized by MALDI mass spectrometry. 
Table S3. Structural information of peptoid-based ladder oligomers subjected to MALDI mass spectrometry and GPC analysis.

\begin{tabular}{|c|c|c|c|c|}
\hline Code & Schematic structure & Formula & $\begin{array}{l}\text { Exact mass } \\
(\mathrm{g} / \mathrm{mol})\end{array}$ & $\begin{array}{l}\text { Expected molecular } \\
\text { weight }\left(M_{\mathrm{n}}\right)(\mathrm{g} / \mathrm{mol})\end{array}$ \\
\hline HB_3 & & $\mathrm{C}_{96} \mathrm{H}_{127} \mathrm{~N}_{15} \mathrm{O}_{20}$ & 1809.938 & 1811.157 \\
\hline HB_ 4 & & $\mathrm{C}_{132} \mathrm{H}_{176} \mathrm{~N}_{20} \mathrm{O}_{28}$ & 2489.296 & 2490.972 \\
\hline HB_ 6 & & $\mathrm{C}_{204} \mathrm{H}_{274} \mathrm{~N}_{30} \mathrm{O}_{44}$ & 3848.013 & 3850.602 \\
\hline VA_Al $\times$ Am_3 $\times 4$ & & $\mathrm{C}_{390} \mathrm{H}_{518} \mathrm{~N}_{60} \mathrm{O}_{82}$ & 7353.821 & 7358.772 \\
\hline VA_Al $\times$ Am_ $4 \times 3$ & & $\mathrm{C}_{390} \mathrm{H}_{518} \mathrm{~N}_{60} \mathrm{O}_{82}$ & 7353.821 & 7358.772 \\
\hline VA_Al $\times$ Am_ $\_\times 6$ & & $\mathrm{C}_{402} \mathrm{H}_{538} \mathrm{~N}_{60} \mathrm{O}_{86}$ & 7581.957 & 7587.060 \\
\hline VA_Al $\times$ Am_6 $\times 4$ & & $\mathrm{C}_{402} \mathrm{H}_{538} \mathrm{~N}_{60} \mathrm{O}_{86}$ & 7581.957 & 7587.060 \\
\hline
\end{tabular}

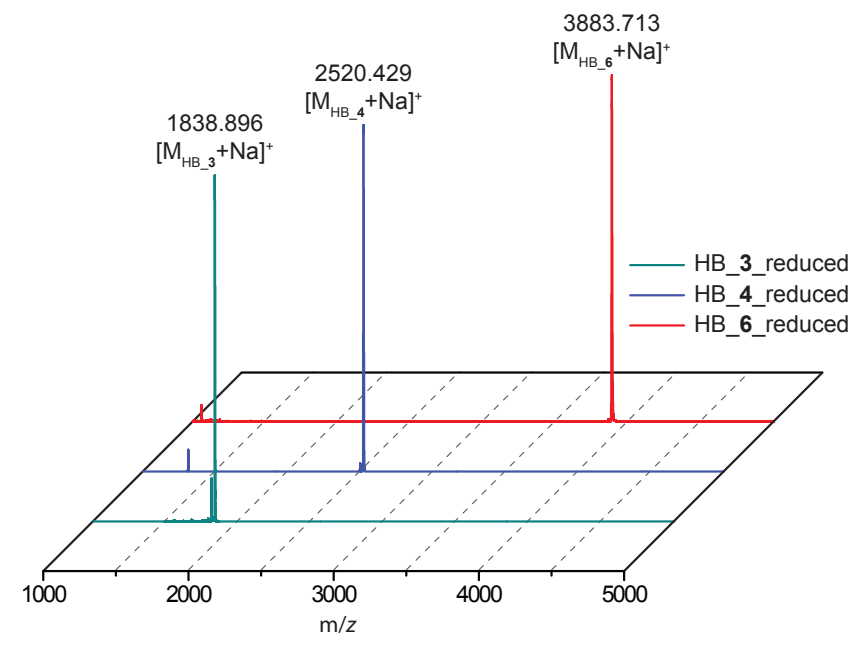

Figure S11. MALDI mass spectra of reduced molecular ladders formed by dynamic covalent oligopeptoid dimerization. Calculated molecular weights: $\left[\mathrm{M}_{\mathrm{HB}_{-} 3^{+N a}}\right]^{+}=1838.974 ;\left[\mathrm{M}_{\mathrm{HB}_{-}}+\mathrm{Na}\right]^{+}=2520.348 ;\left[\mathrm{M}_{\mathrm{HB} \_}{ }^{+N a}\right]^{+}=$ 3883.09 . 


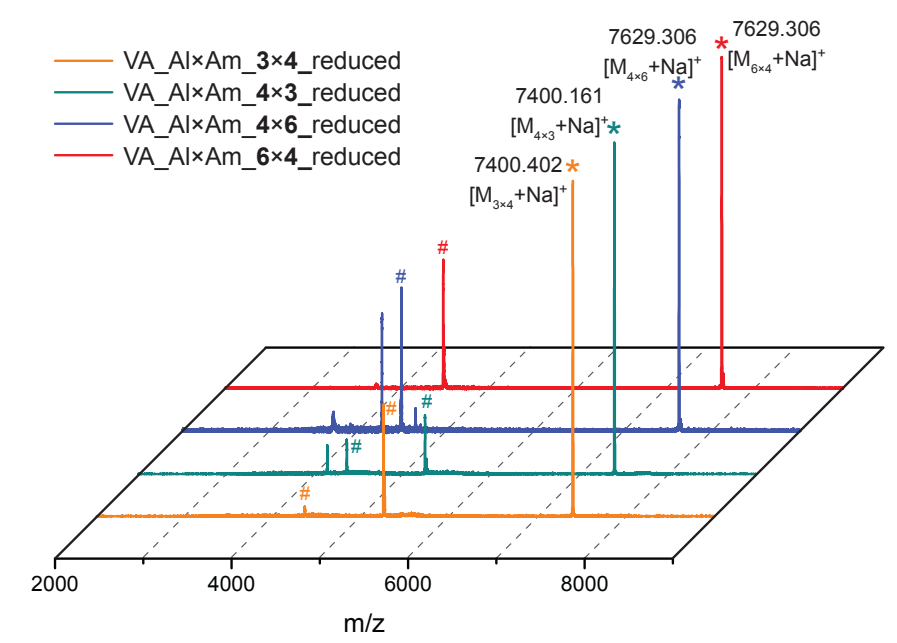

Figure S12. MALDI mass spectra of reduced, Vernier-templated molecular ladders. The target ladder structures are labeled with * symbols and the intermediates are labeled with \#. Calculated molecular weights for the target ladders: $\left[\mathrm{M}_{3 \times 4}+\mathrm{Na}\right]^{+}=\left[\mathrm{M}_{4 \times 3}+\mathrm{Na}\right]^{+}=7400.997 ;\left[\mathrm{M}_{4 \times 6}+\mathrm{Na}\right]^{+}=\left[\mathrm{M}_{6 \times 4}+\mathrm{Na}\right]^{+}=7629.134$. Intermediates are identified as $\left(\mathrm{E}^{3} \mathrm{~A}-\right.$ $\left.\mathrm{Al} \_3\right)_{3}\left(\mathrm{E}^{3} \mathrm{~A}-\mathrm{Am} \_4\right)_{2} ; \quad\left(\mathrm{E}^{3} \mathrm{~A}-\mathrm{Al} \_4\right)_{2}\left(\mathrm{E}^{3} \mathrm{~A}-\mathrm{Am} \_3\right)_{3}, \quad\left(\mathrm{E}^{3} \mathrm{~A}-\mathrm{Al} \_4\right)_{2}\left(\mathrm{E}^{3} \mathrm{~A}-\mathrm{Am} \_3\right)_{2} ; \quad\left(\mathrm{E}^{3} \mathrm{~A}-\mathrm{Al} \_4\right)_{2}\left(\mathrm{E}^{3} \mathrm{~A}-\mathrm{Am} \_6\right)_{1} ; \quad\left(\mathrm{E}^{3} \mathrm{~A}-\right.$ Al_6 $)_{1}\left(\mathrm{E}^{3} \mathrm{~A}-\mathrm{Am} \_4\right)_{2}$. 
Table S4. Structural information of reduced, peptoid-based ladder oligomers subjected to MALDI mass spectrometry analysis.

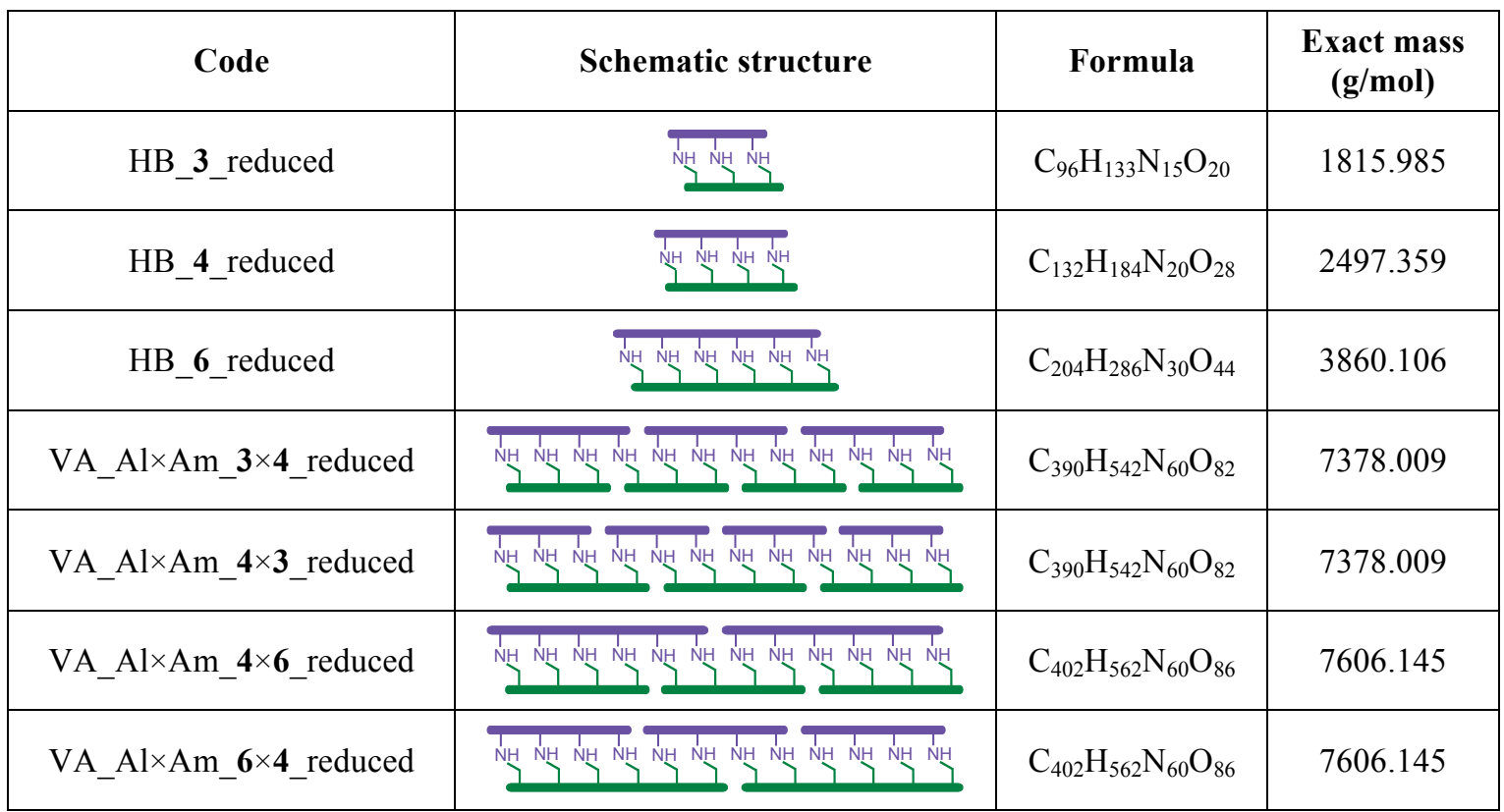

\section{Analytical GPC Calibration Curve}

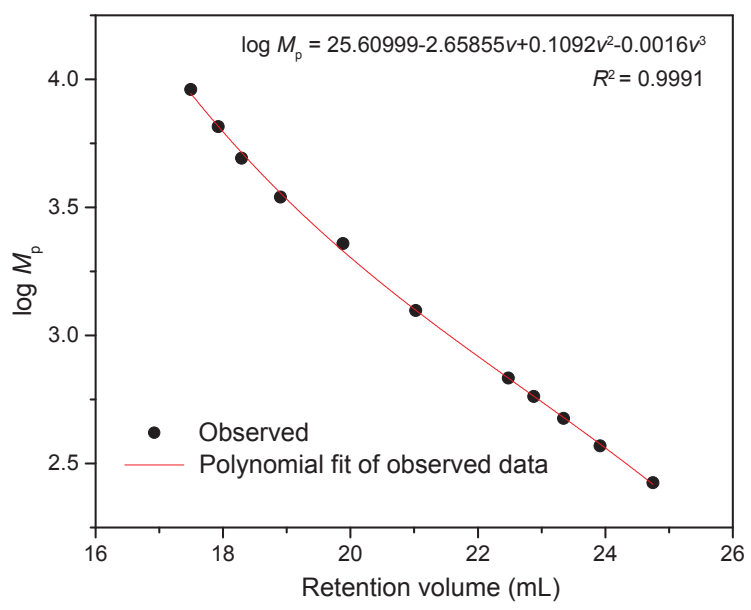

Figure S13. Analytical GPC calibration curve by low dispersity polystyrene standards.

Analytical GPC calibration was performed by running a series of low dispersity polystyrene standards with a molecular weight range from $250 \mathrm{Da}$ to $10 \mathrm{kDa}$ and fitting a third order polynomial curve to the observed $\log M_{\mathrm{p}}$ versus retention volume to generate a calibration curve (see Figure S5). 


\section{Deconvolution of GPC Traces}

GPC traces of peptoid-based ladder oligomers were deconvoluted by fitting Gaussian functions to simulate the peaks using a script written in-house with Matlab R2012a (Mathworks Inc.). In each case, the baseline was subtracted and then 5-6 simulated peaks were utilized to fit the spectra.
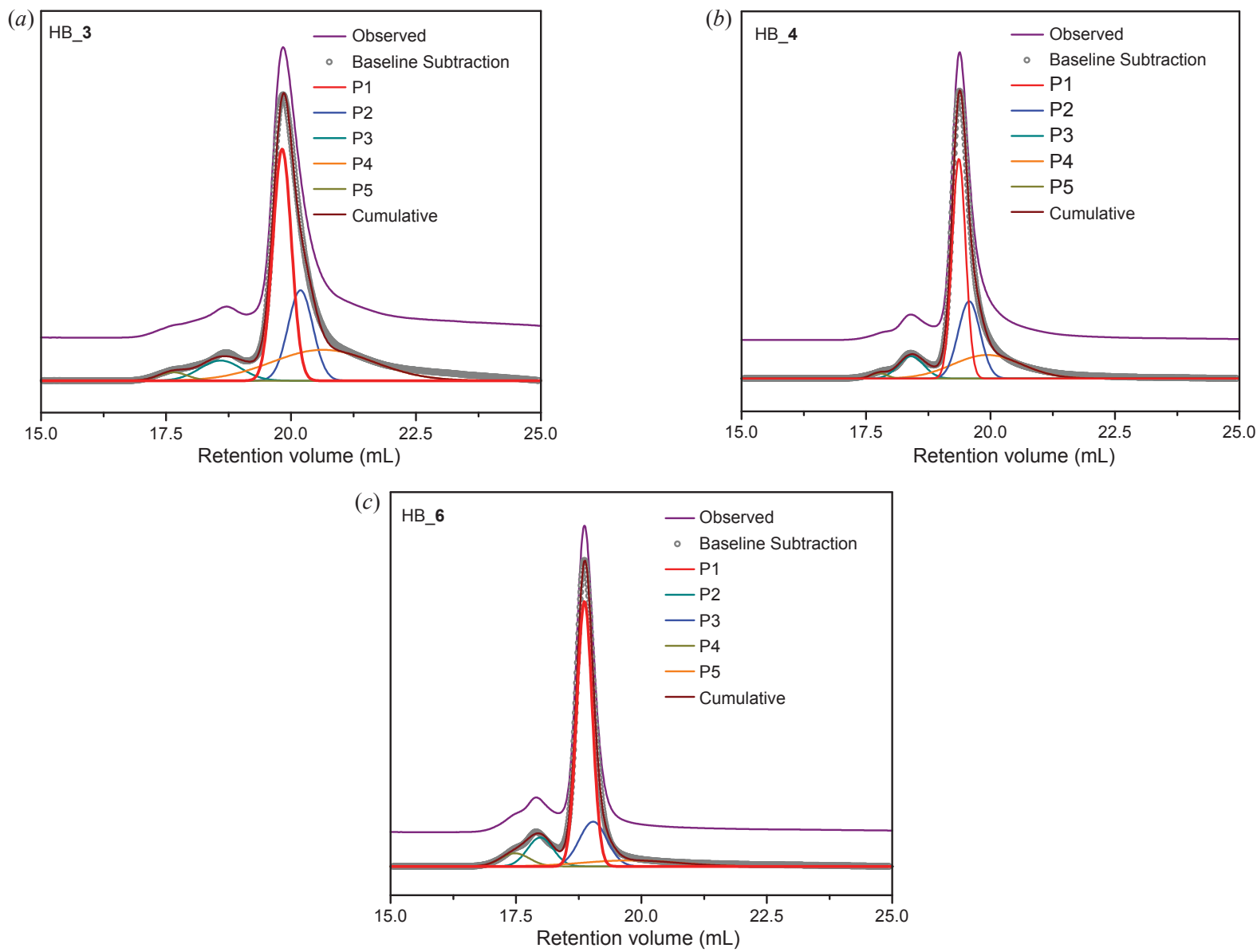

Figure S14. Deconvoluted GPC traces of peptoid-based, dimerized molecular ladders (a) HB_3, (b) HB_4, and (c) HB_6 (straight reaction mixtures). The target product peak is labeled in red.

Table S5. Deconvoluted peak analysis of peptoid-based, dimerized molecular ladders.

\begin{tabular}{|c|c|c|c|c|}
\hline Code & $\boldsymbol{M}_{\mathbf{n}}$ by GPC (g/mol) & Dispersity & Expected $\boldsymbol{M}_{\mathbf{n}}(\mathbf{g} / \mathbf{m o l})$ & Area $(\%)$ \\
\hline HB_3 & 2270 & 1.01 & 1810 & 60.6 \\
\hline HB_4 & 2870 & 1.01 & 2490 & 67.2 \\
\hline HB_6 & 3770 & 1.01 & 3850 & 75.8 \\
\hline
\end{tabular}



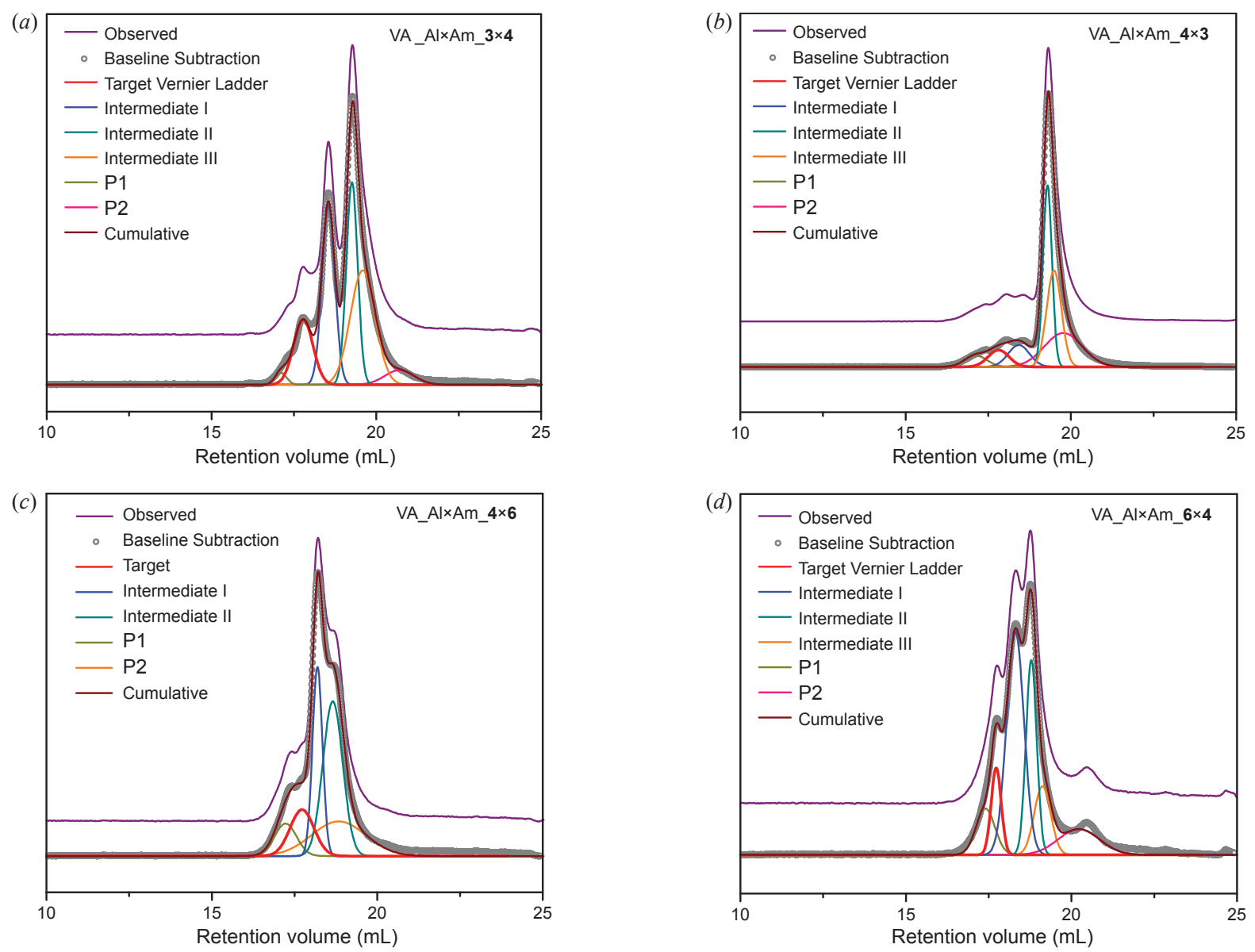

Figure S15. Deconvoluted GPC traces of peptoid-based, Vernier-templated molecular ladders (a) VA_Al $\times$ Am_3 $\times \mathbf{4}$, (b) VA_Al $\times A m \_4 \times 3$, (c) VA_Al $\times A m_{-} \_\times 6$, and (d) VA_Al $\times A m_{-} 6 \times 4$ (straight reaction mixtures). The target product peak is labeled in red. 
Table S6. Deconvoluted peak analysis of VA_Al $\times$ Am_3 $\times \mathbf{4}$.

\begin{tabular}{|c|c|c|c|c|c|}
\hline Product & Schematic structure & $\begin{array}{c}M_{\mathrm{n}} \text { by GPC } \\
(\mathrm{g} / \mathrm{mol})\end{array}$ & Dispersity & Area $(\%)$ & $\begin{array}{r}\text { Expected } \\
M_{\mathrm{n}}(\mathrm{g} / \mathrm{mol})\end{array}$ \\
\hline Target & & 7270 & 1.04 & 13.7 & 7360 \\
\hline Intermediate I & & 4530 & 1.01 & 24.9 & 4320 \\
\hline Intermediate II & & 3030 & 1.01 & 24.8 & 3070 \\
\hline Intermediate III & & 2530 & 1.04 & 30.6 & 2160 \\
\hline
\end{tabular}

Table S7. Deconvoluted peak analysis of VA_Al $\times$ Am_ $4 \times \mathbf{3}$.

\begin{tabular}{|c|c|c|c|c|c|}
\hline Product & Schematic structure & $\begin{array}{c}M_{\mathrm{n}} \text { by GPC } \\
(\mathrm{g} / \mathrm{mol})\end{array}$ & Dispersity & Area $(\%)$ & $\begin{array}{c}\text { Expected } \\
M_{\mathrm{n}}(\mathrm{g} / \mathrm{mol})\end{array}$ \\
\hline Target & & 7120 & 1.05 & 6.5 & 7360 \\
\hline Intermediate I & & 4830 & 1.04 & 8.6 & 4320 \\
\hline Intermediate II & & 2980 & 1.01 & 30.1 & 3080 \\
\hline Intermediate III & & 2680 & 1.01 & 26.4 & 2160 \\
\hline
\end{tabular}

Table S8. Deconvoluted peak analysis of VA_Al $\times$ Am_ $4 \times \mathbf{6}$.

\begin{tabular}{|c|c|c|c|c|c|}
\hline Product & Schematic structure & $\begin{array}{c}M_{\text {n }} \text { by GPC } \\
(\mathrm{g} / \mathrm{mol})\end{array}$ & Dispersity & Area $(\%)$ & $\begin{array}{c}\text { Expected } \\
M_{\mathrm{n}}(\mathrm{g} / \mathrm{mol})\end{array}$ \\
\hline Target & & 7700 & 1.06 & 12.8 & 7590 \\
\hline Intermediate I & $\frac{\mathrm{NH}_{2} \mathrm{NH}_{2} \mathrm{NH}}{4}$ & 5640 & 1.01 & 21.8 & 5170 \\
\hline Intermediate II & & 4220 & 1.03 & 35.4 & 4430 \\
\hline
\end{tabular}


Table S9. Deconvoluted peak analysis of VA_Al $\times$ Am_6 $\times \mathbf{4}$.

\begin{tabular}{|c|c|c|c|c|c|}
\hline Product & Schematic structure & $\begin{array}{c}M_{\text {n }} \text { by GPC } \\
(\mathrm{g} / \mathrm{mol})\end{array}$ & Dispersity & Area (\%) & $\begin{array}{c}\text { Expected } \\
M_{\mathrm{n}}(\mathrm{g} / \mathrm{mol})\end{array}$ \\
\hline Target & & 7650 & 1.01 & 8.8 & 7590 \\
\hline Intermediate I & & 5220 & 1.03 & 39.2 & 5160 \\
\hline Intermediate II & & 3940 & 1.01 & 21.0 & 4440 \\
\hline Intermediate III & & 3250 & 1.02 & 11.4 & 3190 \\
\hline
\end{tabular}




\section{Molecular Ladder Scrambling by Strand Exchange}

Dimerization of aldehyde- and amine-functionalized peptoid oligomers incorporating $\mathrm{ME}^{3} \mathrm{~A}$ as the spacer group (i.e., $\mathrm{ME}^{3} \mathrm{~A}-\mathrm{Al} \_4$ and $\mathrm{ME}^{3} \mathrm{~A}-\mathrm{Am} \_4$ ) was performed using the approach described above to afford a peptoid-based, $\mathrm{ME}^{3} \mathrm{~A}$-bearing molecular ladder $\left(\mathrm{ME}^{3} \mathrm{~A}-\mathrm{HB}\right.$ 4), and the reaction mixture was directly analyzed by MALDI mass (Figure S16) and subsequently utilized for strand exchange studies described below.

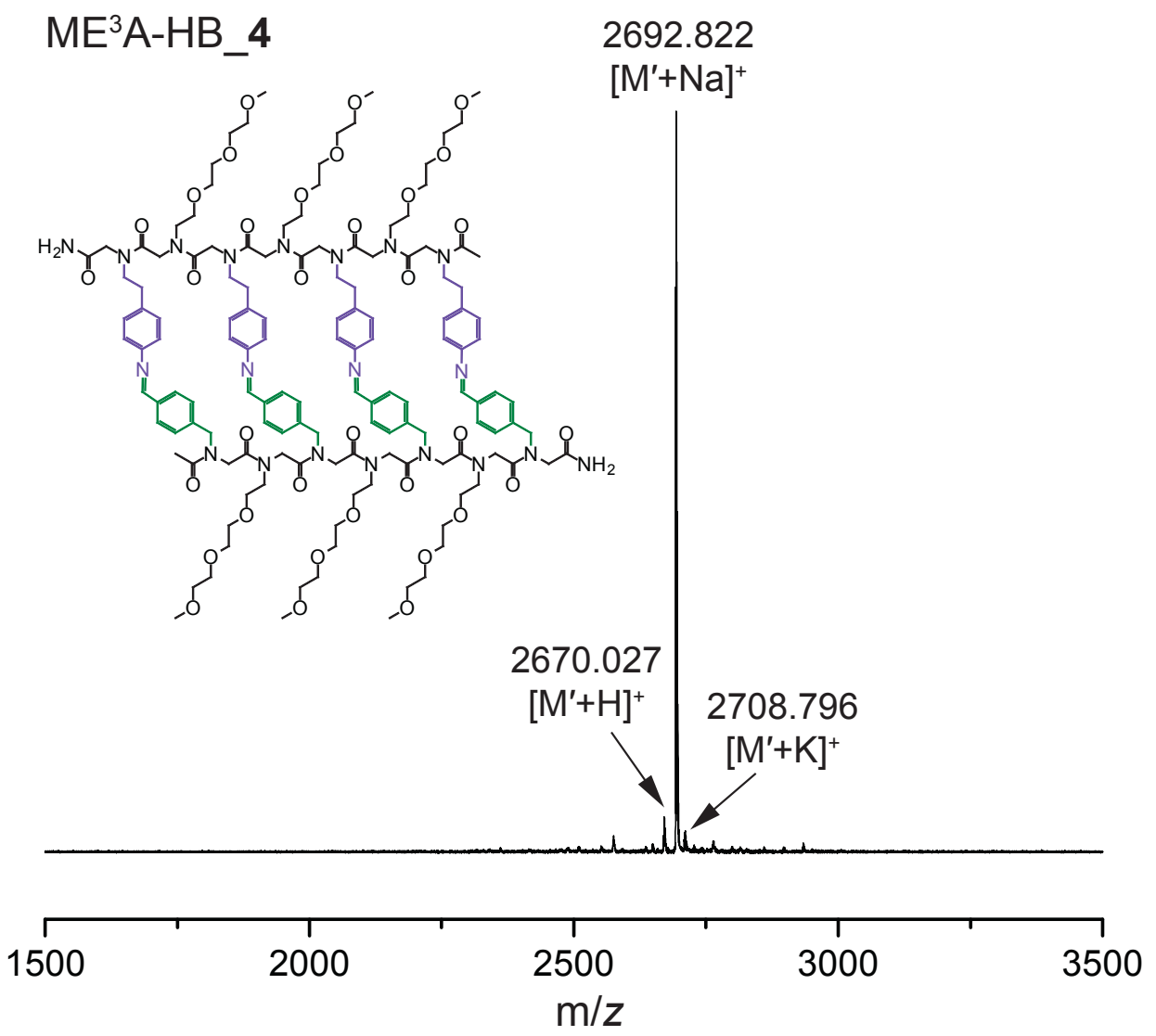

Figure S16. MALDI mass spectrum of molecular ladder formed by dynamic covalent dimerization of oligopeptoids incorporating $\mathrm{ME}^{3} \mathrm{~A}$ spacer. Calculated molecular weights: $\left[\mathrm{M}_{\mathrm{ME}^{3} \mathrm{~A}-\mathrm{HB}} 4+\mathrm{H}\right]^{+}=2670.367,\left[\mathrm{M}_{\mathrm{ME}^{3} \mathrm{~A}-\mathrm{HB}}{ }_{-}+\mathrm{Na}^{+}\right]^{+}=$ 2692.349, $\left[\mathrm{M}_{\mathrm{ME}^{3} \mathrm{~A}-\mathrm{HB}-4}+\mathrm{K}\right]^{+}=2708.323$.

Scrambling between single peptoid strands and molecular ladders were examined by combining $100 \mu \mathrm{L}$ of $10 \mathrm{mM} \mathrm{E}^{3} \mathrm{~A}-\mathrm{Am} \_4$ in $\mathrm{MeCN}$ with $16 \mu \mathrm{L}$ of $10 \mathrm{mM} \mathrm{Sc}(\mathrm{TOf})_{3}$ in $\mathrm{MeCN}$, diluting with $\mathrm{CHCl}_{3}$ to a total volume of $1 \mathrm{~mL}$, and mixing with $1 \mathrm{~mL}$ of a $1 \mathrm{mM}$ solution of the peptoid-based, 4-rung ladder $\mathrm{ME}^{3} \mathrm{~A}$ HB_4, described above, such that the peptoid strand and molecular ladder were present in a 1:1 stoichiometric ratio and the final $\mathrm{Sc}(\mathrm{OTf})_{3}$ concentration was $0.08 \mathrm{mM}$. Scrambling between different molecular ladders was similarly examined by mixing $1 \mathrm{~mL}$ of $1 \mathrm{mM} \mathrm{HB} 4$ (the peptoid-based, 4-rung 
molecular ladder incorporating $\mathrm{E}^{3} \mathrm{~A}$ as the spacer residue) in $\mathrm{CHCl}_{3}$ with $1 \mathrm{~mL}$ of $1 \mathrm{mM} \mathrm{ME} \mathrm{M}^{3} \mathrm{HB} \mathbf{4}^{4}$, both described above, such that the molecular ladders were present in a 1:1 stoichiometric ratio, again with $0.08 \mathrm{mM} \mathrm{Sc}(\mathrm{OTf})_{3}$. Aliquots of the reaction mixture were examined by MALDI mass spectrometry over the course of 9 days to determine the extent of ladder scrambling. Multiple regions of interest were ionized in each sample to obtain an average ratio of target peak intensities, and concentrations of scrambled molecular ladders were estimated by comparing MALDI mass spectrometry signal intensities of the scrambled products with those of the parent molecular ladders.

\section{References}

[1] V. Perron, S. Abbott, N. Moreau, D. Lee, C. Penney, B. Zacharie, Synthesis 2009, 283-289.

[2] O. Ouari, F. Chalier, R. Bonaly, B. Pucci, P. Tordo, J Chem Soc Perk T 2 1998, 2299-2307.

[3] J. Sun, G. M. Stone, N. P. Balsara, R. N. Zuckermann, Macromolecules 2012, 45, 5151-5156.

[4] a) R. J. Simon, R. S. Kania, R. N. Zuckermann, V. D. Huebner, D. A. Jewell, S. Banville, S. Ng, L. Wang, S. Rosenberg, C. K. Marlowe, D. C. Spellmeyer, R. Y. Tan, A. D. Frankel, D. V. Santi, F. E. Cohen, P. A. Bartlett, Proc. Natl. Acad. Sci. U. S. A. 1992, 89, 9367-9371; b) R. N. Zuckermann, J. M. Kerr, S. B. H. Kent, W. H. Moos, J Am Chem Soc 1992, 114, 10646-10647.

[5] C. S. Hartley, E. L. Elliott, J. S. Moore, J Am Chem Soc 2007, 129, 4512-4513. 